\title{
Web-Based Interventions to Help Australian Adults Address Depression, Anxiety, Suicidal Ideation, and General Mental Well-being: Scoping Review
}

Gemma Skaczkowski ${ }^{1}$, BHSc (Hons), PhD; Shannen van der Kruk ${ }^{1}$, BSc, MSc; Sophie Loxton ${ }^{1}$, BPsych (Hons); Donna Hughes-Barton ${ }^{1}$, BSc, MPsych, PhD; Cate Howell ${ }^{2,3}$, BMBS, BAppSc, Dip Clin Hypnosis, MHSM, PhD; Deborah Turnbull ${ }^{4,5}$, BA (Hons), MPsych, PhD; Neil Jensen ${ }^{5}$, MBBS; Matthew Smout ${ }^{6}$, BSc (Hons), MPsych, PhD; Kate Gunn ${ }^{1,5}$, BPsych (Hons), MPsych, PhD

\footnotetext{
${ }^{1}$ Department of Rural Health, Allied Health and Human Performance, University of South Australia, Adelaide, Australia

${ }^{2}$ Australian Medical Placements Health Education and Training, Adelaide, Australia

${ }^{3}$ Torrens University, Adelaide, Australia

${ }^{4}$ School of Psychology, The University of Adelaide, Adelaide, Australia

${ }^{5}$ Freemasons Centre for Male Health and Wellbeing, Adelaide, Australia

${ }^{6}$ Justice and Society, University of South Australia, Adelaide, Australia
}

\section{Corresponding Author:}

Kate Gunn, BPsych (Hons), MPsych, PhD

Department of Rural Health, Allied Health and Human Performance

University of South Australia

North Terrace

Adelaide, 5001

Australia

Phone: 61417852537

Email: kate.gunn@unisa.edu.au

\section{Abstract}

Background: A large number of Australians experience mental health challenges at some point in their lives. However, in many parts of Australia, the wait times to see general practitioners and mental health professionals can be lengthy. With increasing internet use across Australia, web-based interventions may help increase access to timely mental health care. As a result, this is an area of increasing research interest, and the number of publicly available web-based interventions is growing. However, it can be confusing for clinicians and consumers to know the resources that are evidence-based and best meet their needs.

Objective: This study aims to scope out the range of web-based mental health interventions that address depression, anxiety, suicidal ideation, or general mental well-being and are freely available to Australian adults, along with their impact, acceptability, therapeutic approach, and key features.

Methods: The PRISMA (Preferred Reporting Items for Systematic Reviews and Meta-Analyses) guidelines for scoping reviews (PRISMA-ScR [PRISMA extension for Scoping Reviews]) guided the review process. Keywords for the search were depression, anxiety, suicide, and well-being. The search was conducted using Google as well as the key intervention databases Beacon, Head to Health, and e-Mental Health in Practice. Interventions were deemed eligible if they targeted depression, anxiety, suicidal ideation, or general mental well-being (eg, resilience) in adults; and were web-based, written in English, interactive, free, and publicly available. They also had to be guided by an evidence-based therapeutic approach.

Results: Overall, 52 eligible programs were identified, of which 9 (17\%) addressed depression, 15 (29\%) addressed anxiety, $13(25 \%)$ addressed general mental well-being, and $13(25 \%)$ addressed multiple issues. Only 4\% (2/52) addressed distress in the form of suicidal ideation. The most common therapeutic approach was cognitive behavioral therapy. Half of the programs guided users through exercises in a set sequence, and most programs enabled users to log in and complete the activities on their own without professional support. Just over half of the programs had been evaluated for their effectiveness in reducing symptoms, and $11 \%(6 / 52)$ were being evaluated at the time of writing. Program evaluation scores ranged from $44 \%$ to $100 \%$, with a total average score of $85 \%$. 
Conclusions: There are numerous web-based programs for depression, anxiety, suicidal ideation, and general well-being, which are freely and publicly available in Australia. However, identified gaps include a lack of available web-based interventions for culturally and linguistically diverse populations and programs that use newer therapeutic approaches such as acceptance and commitment therapy and dialectical behavior therapy. Despite most programs included in this review being of good quality, clinicians and consumers should pay careful attention when selecting which program to recommend and use, as variations in the levels of acceptability and impact of publicly available programs do exist.

(JMIR Ment Health 2022;9(2):e31018) doi: $\underline{10.2196 / 31018}$

\section{KEYWORDS}

web-based interventions; depression; anxiety; suicide; well-being; mental health; technology; access to health care

\section{Introduction}

\section{Background}

Approximately half of the Australian population experiences a common mental disorder (eg, anxiety and depression) at some point in their lives [1]. Mental and substance use disorders are the fourth highest contributor to total disease burden after cancer, cardiovascular disease, and musculoskeletal conditions [2], whereas suicide is the leading cause of death among young people aged between 15 and 44 years and the fourth leading cause of death in those aged between 45 and 64 years [2]. Despite these alarming statistics, only one-third of people experiencing mental illness will access support services [3]. The reasons for this may be structural (eg, availability of suitable services), knowledge-related (eg, limited awareness of the benefits of accessing mental health services), or attitudinal (eg, stigma and stoicism).

When people do seek help for mental health issues, $71 \%$ will contact their general practitioner (GP) at the first instance [3]. GPs play a crucial role in suicide prevention; approximately $45 \%$ of people who die by suicide have had contact with primary care in the preceding month and 77\% in the preceding year [4]. However, GPs report the lack of tools needed to administer psychological care, including a lack of time, resources, and confidence [5]. Although GPs and their patients increasingly value psychological support, a shortage of professionals and time on wait lists, averaging between 2 and 6 months, is both frustrating and distressing for GPs and their patients.

There is a pressing need to bridge the gap between primary health and mental health care, particularly for Australians facing greater difficulties in accessing mental health care and who are at particular risk of suicide, such as those in rural areas. Fortunately, Australians are increasingly willing to use the internet to search for health-related information [6], with at least $86 \%$ of Australian households now having access to the internet [7]. Therefore, web-based resources and interventions may supplement face-to-face services, providing support to people as they wait for appointments [8] and new avenues to reach those who face structural or attitudinal barriers to accessing face-to-face mental health services [9]. Web-based resources have the advantage of enabling consumers' anonymity and access at any time of the day or night, from the privacy and convenience of their own homes [8].

Across the globe, web-based interventions have been shown to effectively reduce the severity of symptoms of depression [10], anxiety [11], and social anxiety [12] and increase mental health literacy [13] and the ability to recognize, accept, deal with, and help prevent mental health issues [14]. The quality of this evidence is high, with a number of randomized controlled trials (RCTs; eg, the studies by McDermott et al [10], Powell et al [12], and Kiropoulos et al [13]) and systematic reviews (eg, the studies by Renton et al [15] and Ashford et al [16]) illustrating their value. Participation has also been associated with decreases in personal stigmatizing attitudes toward depression in the mainstream population [17] and among immigrants [13], as well as decreases in stigmatizing attitudes toward suicide [18] and help seeking [19]. Importantly, web-based interventions have been identified as acceptable in difficult-to-reach populations such as farmers [20], young people [21], and culturally and linguistically diverse groups [22,23]. They can be successfully integrated into routine primary care [24,25], and many Australian GPs support the notion of aiding their patients' mental well-being through these resources [26]. In addition, web-based interventions have been found to be cost-effective for development and delivery; many are offered for free to consumers [8]. However, with the plethora of interventions emerging on the internet [27], there is a need for reliable and clear recommendations to help clinicians and consumers select web-based interventions that are based on evidence-based therapeutic approaches and meet their needs. The e-Mental Health in Practice (eMHPrac) project, funded by the Australian Government, has a range of resources to help Australian health practitioners find digital mental health programs for use with their patients [28]. This provides an excellent quick reference guide for clinicians. However, it does not outline the types of therapeutic approaches and tools used or indicate whether resources are evidence-based.

A total of 2 existing international scoping reviews summarize the characteristics of web-based interventions available for the treatment of depression in Canada in 2014 [15] and anxiety in the United Kingdom in 2016 [16]. The review of programs for depression by Renton et al [15] identified 32 programs, with only 12 having published evidence of efficacy. Furthermore, the review of programs for anxiety by Ashford et al [16] identified 34 programs, with only 17 having published evidence of efficacy. Given the rapid creation of web-based mental health resources in recent years [27] and the tendency for web-based mental health programs to change, arise, and disappear with the provision or withdrawal of funding, there is a need for updated information on interventions that consumers and clinicians are likely to come across via search engines in the Australian context. Of particular benefit to clinicians would be a focus on 
free and publicly available interventions, based on evidence-based therapeutic approaches, that Australians can immediately find or be referred to. Clinicians have also indicated that it would be useful to include web-based interventions for adults seeking help for general mental well-being and suicidal ideation, which are issues commonly encountered in clinical practice but not covered by past reviews. We note that many reviews of the evidence for web-based interventions for specific disorders (eg, depression [29-31]) and for specific populations (eg, adolescents and young adults [32,33]) have been undertaken in recent years. Although these are helpful for researchers and intervention developers, they are less likely to meet the practical information needs of consumers and clinicians, as many of the interventions contained in the reviews are not available outside research settings, and others that are publicly accessible, have not been evaluated and therefore are not included in the reviews.

\section{Objectives}

The purpose of this research is to identify the scope of and evidence for web-based mental health interventions addressing depression, anxiety, suicidal ideation, or general mental well-being that are currently available for free to Australian adults, and in doing so, develop a quick reference guide for GPs and mental health clinicians. It seeks to answer the following questions [34]:

1. What web-based interventions addressing depression, anxiety, suicidal ideation, or general mental well-being are currently freely available (ie, at no cost and open to Australians) in Australia?

2. What are the key gaps?

3. What are the characteristics, including evidence of impact, credibility, and accessibility, of the interventions that are available?

\section{Methods}

This scoping review was conducted in accordance with the PRISMA-ScR (Preferred Reporting Items for Systematic Reviews and Meta-Analyses extension for Scoping Reviews) [35]. The study protocol was registered in the Open Science Framework [36].

\section{Eligibility Criteria}

Eligible interventions were those that (1) targeted depression, anxiety, suicidal ideation, or general mental well-being (eg, resilience and stress); (2) were web-based; (3) had an interactive component (ie, the delivery of content was not entirely in a passive manner); (4) were designed for adults; (5) were free (ie, at no cost) and publicly available (including via registration, application, or referral from a health care professional); (6) were available in English; and (7) were based on an evidence-based therapeutic approach.
Interventions were excluded if (1) they were targeted at consumers with a primary general medical condition (eg, survivors of cancer); (2) solely provided reading materials on one of the inclusion outcomes (ie, psychoeducation) or only offered a single tool (eg, diary-keeping, mood-monitoring, and meditation) without relevant psychoeducation or explanation of a broader evidence-based therapeutic approach; (3) were targeted at health professionals for training purposes; (4) were part of a research project that restricted access or limited program availability; or (5) included classes that had to be attended in person.

\section{Search Strategy}

A search was conducted in October 2020 for key terms using Google (Australian version). As the previously published scoping review on web-based interventions for depression only found 1 additional website when searching Yahoo and Bing [15], only Google was searched for this review. A total of 4 searches were conducted by searching the following keywords: depression, anxiety, suicide, and wellbeing. A search log was kept to record the number of hits for each search, as well as the number of websites that were included or excluded (Multimedia Appendix 1). The search was terminated for the terms after searching the first 10 pages of results (ie, approximately 100 hits per keyword). Cookies and existing search histories were deleted before the start of each search, and the search results were downloaded into a Microsoft Excel file using a Chrome extension (ie, Export Search Results).

In addition, key intervention databases were searched by 2 reviewers (SvdK and SL). These included Beacon [37], Head to Health [38], and eMHPrac websites [39]. Finally, the reference lists and programs listed in the 2 existing scoping reviews on web-based depression [15] and anxiety [16] resources were searched by 2 reviewers (SvdK and SL) to identify any programs not included in the searches outlined. An overview of program developers and website links is provided in Multimedia Appendix 2.

\section{Selection of Web-Based Programs}

Screening of the search results to identify eligible web-based programs was completed in 2 stages. The first stage involved screening all search hyperlinks by 1 reviewer (SvdK) to eliminate websites that solely provided information on 1 of the outcomes or those that were clearly irrelevant (eg, mental health information only websites, YouTube videos, or blogs). The remainder of the websites was then divided into those that directly offered web-based programs and those that linked to web-based programs. Any duplicate websites were removed. The second stage involved screening potential eligible programs against the eligibility criteria by 2 reviewers (SvdK and SL; Figure 1). 
Figure 1. PRISMA (Preferred Reporting Items for Systematic Reviews and Meta-Analyses) flow diagram of program selection.

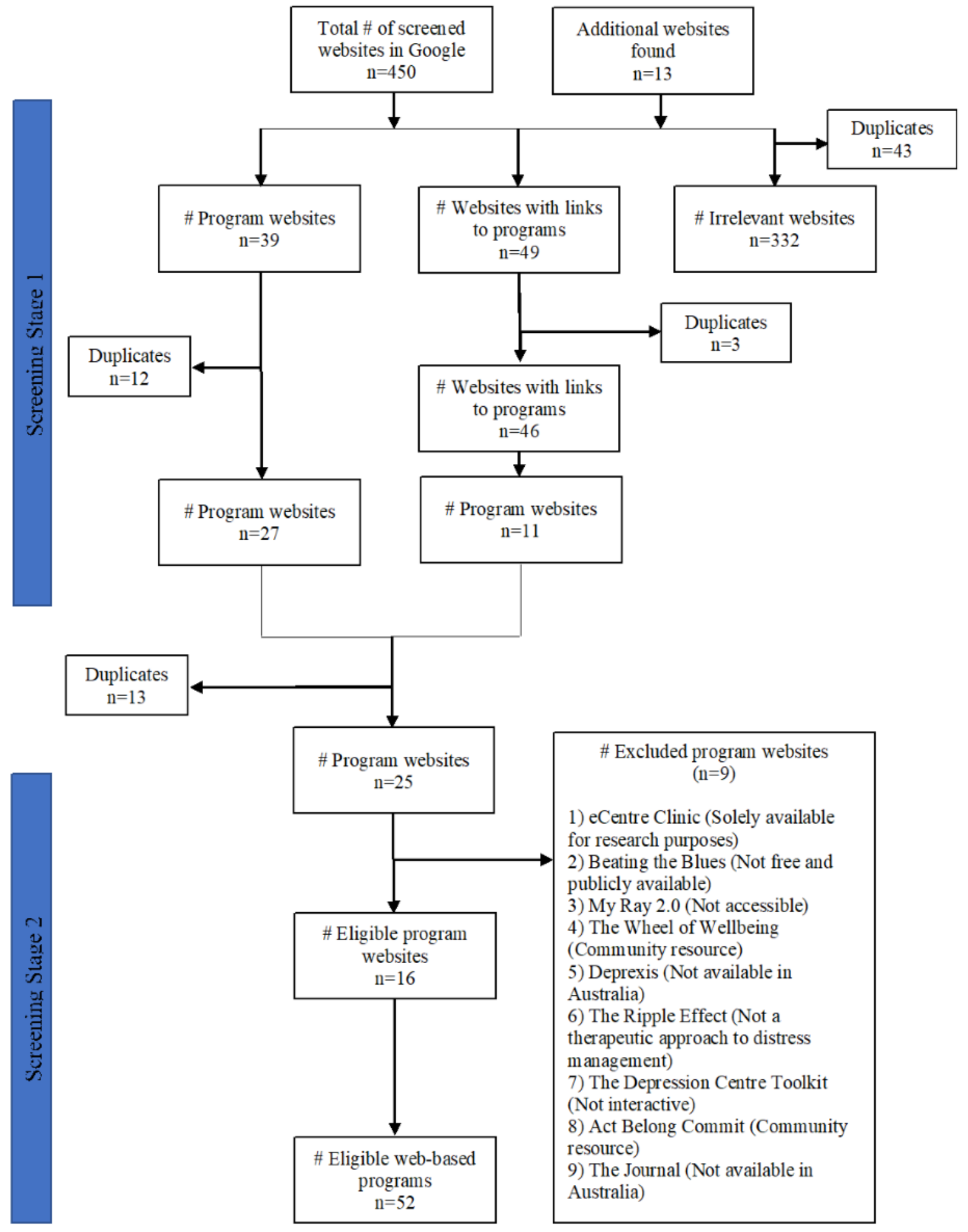

\section{Data Extraction}

A data extraction form was created to systematically evaluate each identified program. The extraction tool was adapted from a previously published framework designed to evaluate and report internet intervention studies [40]. This framework has been used in 2 recent scoping reviews examining web-based interventions for depression [15] and anxiety [16]. The main

categories and subcategories of investigation were adapted from these studies (Table 1).

Websites were trialed and data extraction was undertaken by 3 reviewers (SvdK, SL, and GS) in November to December 2020, with the reviewers independently extracting data for each website so that the data extraction was performed in duplicate. Discrepancies in coding were resolved by discussion and, where necessary, investigated by the third reviewer. Where necessary, program authors were contacted to request access to the 
program. For 2 websites (MindSpot and Evolution Health), information was extracted from the public-facing pages where possible, and the remaining information was requested from the website authors or developers because of time and administrative constraints.

Table 1. Data extraction categories.

\begin{tabular}{ll}
\hline Main categories and subcategories & Evaluation focus \\
\hline Website characteristics & \\
Accessibility and credibility & - How is the website accessed (free, via assessment, or via referral from a health care professional)? \\
& - Mobile phone rendering (yes or no) \\
& - Advertisements (if yes, relevant or irrelevant?) \\
Authorship details & - Presented contact details of authors or developers? (yes or no)
\end{tabular}

\section{Program characteristics}

Focus and target population

Professional support

Other support

Program interactivity

Multimedia channel of delivery

Degree of synchronicity

Audience reach

\section{Intervention characteristics}

Model of change

Type and dose of intervention

Intervention features

\section{Empirical evidence}

Program evaluation
- $\quad$ Target issue

- Target audience

- Therapist support (within the intervention, ability to link independent clinicians with the program)

- $\quad$ Peer support (did the program offer peer support, eg, forums? Was this monitored)?

- This facet is addressed by type and dose of intervention

- Presentation format or mode of delivery (eg, how the content was delivered; text, audio, or video offered; use of character examples; and case scenarios)?

- Were email or SMS text message reminders or follow-up offered?

- This facet is addressed in focus and target population

- $\quad$ Therapeutic approach

- Was the program structured or unstructured?

- Number of modules

- Suggested or set treatment length

- Self-assessments (were these mandatory or optional)?

- Worksheets (yes or no)

- Mood or symptom monitoring (yes or no)

- Diary (yes or no)

- Forums (yes or no)

- Other features (if yes, specify)
- Usability (did the program provide users with statistics on registered users, completion rates, attrition rates? Testimonial videos or case studies?)

- Empirical evidence (searched program website and Beacon ${ }^{\mathrm{a}}$ database and contacted authors)

- $\quad$ Type of evidence (eg, $\mathrm{RCT}^{\mathrm{b}}$, pre, or post?)

\section{Ethical issues}
Website
- $\quad$ Privacy notice specified?
- Terms and conditions specified?
Emergency
- Does the program offer links to crisis or emergency contacts? 
To identify relevant peer-reviewed papers and thereby explore the empirical evidence behind each program, we checked the program websites and the Beacon directory and contacted the program authors. A total of 3 reviewers (SvdK, DHB, and GS) then conducted a rapid review of the peer-reviewed evidence for each program. This was limited to evaluations of the complete web-based program rather than individual program elements or individual modules. In addition, the level of evidence was determined for each peer-reviewed study [41].

\section{Evaluation of Programs}

A program evaluation scoring system was adapted from previously published guidelines for evaluating and reporting web-based intervention research [40], similar to Ashford et al [16]. The scale comprised 17 yes or no closed-ended questions (Textbox 1). Questions that were answered yes were given a score of 1 and questions that were answered no were scored 0 . Questions that could not be evaluated were given a score of 0 . Questions that were not relevant to the website were excluded from the final score (listed as "NA"). The final scores were calculated as a percentage of the number of relevant items for that website. A total of 3 reviewers (SvdK, SL, and GS) independently assessed the programs against the evaluation scoring system. Discrepancies were resolved through discussion.

Textbox 1. Program evaluation criteria (subcategories and questions).

Authorship details

- Were the names and credentials of authors or organizations present?

- Were contact details provided?

- Was country of origin stated?

Focus and target population

- Was the primary focus, goals, or objectives of the intervention stated?

- Was the target audience or mental health issue defined?

\section{Professional support}

- Was there a statement of professional support (ie, clinicians involved in website development)?

Multimedia channel of delivery

- Did the program offer a multimedia content delivery (ie, a combination of text, video, graphics, and audio formats)?

\section{Model of change}

- Was the model of change (ie, type of therapy used) defined or stated?

\section{Type and dose of intervention}

- Were the number of modules or time to complete each module stated?

- Was the intervention tailored to the user or was it generic for all users (ie, was the program content individualized with username, characteristics, previous responses)?

- Was the program easy to navigate (eg, clear links to the home page and easily able to stop or start the program)?

- Was the information on what is covered in the intervention modules provided (ie, names of modules or a short description)?

\section{Program evaluation}

- Was evidence for the program provided to the user (ie, attrition data, success rate, completion rate, or number of users in the program or testimonials)?

- Has the program been empirically evaluated?

\section{Ethical issues}

- Was a unique username or password provided to users?

- Was a privacy notice specified?

- Were the terms and conditions specified? 


\section{Synthesis of Results}

The results were summarized quantitatively by main categories and subcategories, as displayed in Table 1. Where possible, descriptive statistics were used to summarize these findings.

\section{Results}

\section{Selection of Web-Based Programs}

As seen in Figure 1, 450 websites were screened through Google search, and an additional 13 websites were identified through database searches. Approximately 10.6\% (49/463) of websites with links to potentially eligible programs and 39 potentially eligible programs were identified. Of the 49 websites, $25(51 \%)$ program websites were screened for eligibility, of which 16 (64\%) websites (leading to 52 programs) were included in the review. Of the 52 programs, 20 (39\%) programs were classified as web-based interactive, and $32(62 \%)$ programs were classified as web-based with downloadable worksheets/resources. These 2 types of programs were separated in the summary tables.

We only found 2 programs (BeyondNow and My Digital Health iConsider Life) designed to address distress in the context of suicidal ideation. It should be noted that the inclusion of BeyondNow was debated between team members, as we experienced difficulty in determining whether it fully fulfills the criteria of using an evidence-based therapeutic approach. Nonetheless, it is considered a valuable tool for the management of suicide ideation; therefore, it was included in this review.

\section{Website Characteristics}

\section{Accessibility and Credibility}

Of the 52 programs, $36(69 \%)$ were available at no cost and open to all Australians, 9 (17\%) were accessible via referral from a health care professional (via This Way Up), 4 (8\%) required an assessment before registration (via MindSpot), and $3(6 \%)$ required sign-up to a research project (via My Digital Health; Tables 2 and 3). A free website (with 5/52, 10\% programs) provided users with access to the basic course content without payment but required a paid upgrade to access additional features (Living Life to the Full). In total, 8\% (4/52) of courses from Evolution Health were free to users. In addition, Evolution Health is a technology provider that licenses paid white label versions of its platform to organizations that contain only the course that the organization wants (eg, the smoking cessation course), thereby creating a tailored version of the platform for those organizations to distribute freely to their members.

Registration was required by $83 \%$ (43/52) of programs and most often required users' names, emails, location (country, city, or postcode), and age. Approximately 6\% (3/52) of programs created by My Digital Health were offered as part of a research study; of these, 2 programs, Life Flex and Life Flex-LGBQ (lesbian, gay, bisexual, and queer), required the completion of comprehensive demographic, mental health, and well-being questionnaires before access was granted. Approximately $17 \%$ (9/52) of programs did not require registration and allowed users anonymous access. All websites were accessible via mobile phones and did not contain advertisements, except for the 5 Living Life to the Full programs, which contained advertisements for e-books related to the course content. 
Table 2. Program characteristics of web-based interactive programs.

\begin{tabular}{|c|c|c|c|c|}
\hline Program & $\begin{array}{l}\text { Focus and target population } \\
\text { (if specified) }\end{array}$ & Access & Therapist support & Presentation format \\
\hline \multicolumn{5}{|l|}{ Depression } \\
\hline $\begin{array}{l}\text { eCouch-Depres- } \\
\text { sion Program }\end{array}$ & Depression, $\geq 16$ years & Free & No & $\begin{array}{ll}\text { - } & \text { Text-based } \\
\text { - } & \text { Graphics } \\
\text { - } & \text { Characters or case scenar- } \\
\text { - } & \text { Aus } \\
\text { - } & \text { Comic slideshow format }\end{array}$ \\
\hline $\begin{array}{l}\text { Mum2B-Mood- } \\
\text { Booster }\end{array}$ & $\begin{array}{l}\text { Depression during pregnan- } \\
\text { cy, expectant mothers }\end{array}$ & Free & No & $\begin{array}{ll}\text { - } & \text { Text-based } \\
\text { - } & \text { Graphics } \\
\text { - } & \text { Audio or video }\end{array}$ \\
\hline $\begin{array}{l}\text { MumMood-Boost- } \\
\text { er }\end{array}$ & $\begin{array}{l}\text { Postnatal depression, wom- } \\
\text { en with postnatal depression }\end{array}$ & Free & Yes, optional & $\begin{array}{ll}\text { - } & \text { Text-based } \\
\text { - } & \text { Graphics } \\
\text { - } & \text { Audio or video }\end{array}$ \\
\hline $\begin{array}{l}\text { OnTrack-Depres- } \\
\text { sion }\end{array}$ & Depression, $\geq 18$ years & Free & No & $\begin{array}{ll}\text { - } & \text { Text-based } \\
\text { - } & \text { Graphics } \\
\text { - } & \text { Audio or video }\end{array}$ \\
\hline \multicolumn{5}{|l|}{ Anxiety } \\
\hline $\begin{array}{l}\text { eCouch-Anxiety } \\
\text { and Worry Program }\end{array}$ & $\begin{array}{l}\text { Anxiety and worry, } \geq 16 \\
\text { years }\end{array}$ & Free & No & $\begin{array}{ll}\text { - } & \text { Text-based } \\
\text { - } & \text { Graphics } \\
& \text { Characters or case scenar- } \\
\text { - } & \text { Audio or video } \\
\text { - } & \text { Comic slideshow } \\
\text { - } & \text { Format }\end{array}$ \\
\hline
\end{tabular}

\section{Multi-issue}

\begin{tabular}{|c|c|c|c|}
\hline $\begin{array}{l}\text { MindSpot-Indige- } \\
\text { nous Wellbeing }\end{array}$ & $\begin{array}{l}\text { Managing depression and } \\
\text { anxiety, Aboriginal and } \\
\text { Torres Strait Islanders, } \geq 18 \\
\text { years }\end{array}$ & $\begin{array}{l}\text { Free following mandatory } \\
\text { assessment and contact with } \\
\text { MindSpot therapist }\end{array}$ & $\begin{array}{l}\text { Yes, optional access to } \\
\text { MindSpot therapist }\end{array}$ \\
\hline $\begin{array}{l}\text { MindSpot-Mood } \\
\text { Mechanic }\end{array}$ & $\begin{array}{l}\text { Managing depression and } \\
\text { anxiety, young adults, 18-25 } \\
\text { years }\end{array}$ & $\begin{array}{l}\text { Free following mandatory } \\
\text { assessment and contact with } \\
\text { MindSpot therapist }\end{array}$ & $\begin{array}{l}\text { Yes, optional access to } \\
\text { MindSpot therapist }\end{array}$ \\
\hline $\begin{array}{l}\text { MindSpot-Wellbe- } \\
\text { ing }\end{array}$ & $\begin{array}{l}\text { Managing depression and } \\
\text { anxiety, 26-65 years }\end{array}$ & $\begin{array}{l}\text { Free following mandatory } \\
\text { assessment and contact with } \\
\text { MindSpot therapist }\end{array}$ & $\begin{array}{l}\text { Yes, optional access to } \\
\text { MindSpot therapist }\end{array}$ \\
\hline $\begin{array}{l}\text { MindSpot-Wellbe- } \\
\text { ing Plus }\end{array}$ & $\begin{array}{l}\text { Managing depression and } \\
\text { anxiety, } \geq 60 \text { years }\end{array}$ & $\begin{array}{l}\text { Free following mandatory } \\
\text { assessment and contact with } \\
\text { MindSpot therapist }\end{array}$ & $\begin{array}{l}\text { Yes, optional access to } \\
\text { MindSpot therapist }\end{array}$ \\
\hline MoodGym & Anxiety and depression & Free & No \\
\hline MyCompass & $\begin{array}{l}\text { Mild to moderate depres- } \\
\text { sion, anxiety and stress, } \geq 18 \\
\text { years }\end{array}$ & Free & No \\
\hline
\end{tabular}

- Text-based

- Graphics

- Audio or video

- Text-based

- Graphics

- Audio or video

- Text-based

- Graphics

- Audio or video

- Text-based

- Graphics

- Audio or video

- Text-based

- Graphics

- Audio or video

- Text-based

- Graphics

- Audio or video 


\begin{tabular}{|c|c|c|c|c|}
\hline Program & $\begin{array}{l}\text { Focus and target population } \\
\text { (if specified) }\end{array}$ & Access & Therapist support & Presentation format \\
\hline $\begin{array}{l}\text { My Digital } \\
\text { Health-Life Flex }\end{array}$ & $\begin{array}{l}\text { Anxiety or depression, } \geq 18 \\
\text { years }\end{array}$ & $\begin{array}{l}\text { Free (via research study } \\
\text { participation) }\end{array}$ & $\begin{array}{l}\text { Yes, ability to connect inde- } \\
\text { pendent clinician to program }\end{array}$ & $\begin{array}{ll}\text { - } & \text { Text-based } \\
\text { - } & \text { Graphics } \\
\text { - } & \text { Audio or video }\end{array}$ \\
\hline $\begin{array}{l}\text { My Digital } \\
\text { Health-Life Flex } \\
\text { LGBQ }^{\mathrm{a}}\end{array}$ & $\begin{array}{l}\text { Anxiety or depression, } \geq 18 \\
\text { years who are part of the } \\
\text { LGBQ community }\end{array}$ & $\begin{array}{l}\text { Free (via research study } \\
\text { participation) }\end{array}$ & $\begin{array}{l}\text { Yes, ability to connect inde- } \\
\text { pendent clinician to program }\end{array}$ & $\begin{array}{ll}\text { - } & \text { Text-based } \\
\text { - } & \text { Graphics } \\
\text { - } & \text { Audio or video }\end{array}$ \\
\hline \multicolumn{5}{|l|}{ General well-being } \\
\hline $\begin{array}{l}\text { eCouch-Bereave- } \\
\text { ment and Loss Pro- } \\
\text { gram }\end{array}$ & $\begin{array}{l}\text { Bereavement and loss, } \geq 16 \\
\text { years }\end{array}$ & Free & No & $\begin{array}{ll}\text { - } & \text { Text-based } \\
\text { - } & \text { Graphics } \\
\text { - } & \text { Characters or case scenar- } \\
\text { - } & \text { ios } \\
\text { - } & \text { Audio or video } \\
& \text { Comic slideshow format }\end{array}$ \\
\hline $\begin{array}{l}\text { eCouch-Divorce } \\
\text { and separation }\end{array}$ & $\begin{array}{l}\text { Divorce and separation, } \geq 16 \\
\text { years }\end{array}$ & Free & No & $\begin{array}{ll}\text { - } & \text { Text-based } \\
\text { - } & \text { Graphics } \\
\text { - } & \text { Characters or case scenar- } \\
\text { - } & \text { ios } \\
\text { Comic slideshow format }\end{array}$ \\
\hline ifarmwell & $\begin{array}{l}\text { Well-being, farmers, } \geq 18 \\
\text { years }\end{array}$ & Free & No & $\begin{array}{ll}\text { - } & \text { Text-based } \\
\text { - } & \text { Graphics } \\
\text { - } & \text { Audio or video }\end{array}$ \\
\hline The Desk & $\begin{array}{l}\text { Well-being, Australian ter- } \\
\text { tiary students }\end{array}$ & Free & No & $\begin{array}{ll}\text { - } & \text { Text-based } \\
\text { - } & \text { Graphics } \\
\text { - } & \text { Audio or video }\end{array}$ \\
\hline \multicolumn{5}{|l|}{ Suicidal ideation } \\
\hline $\begin{array}{l}\text { My Digital } \\
\text { Health-iConsider- } \\
\text { Life }\end{array}$ & $\begin{array}{l}\text { Decision support crisis digi- } \\
\text { tal health program, } \geq 18 \\
\text { years }\end{array}$ & $\begin{array}{l}\text { Free (via research study } \\
\text { participation) }\end{array}$ & No & $\begin{array}{ll}\text { - } & \text { Text-based } \\
\text { - } & \text { Audio or video }\end{array}$ \\
\hline BeyondNow & Suicide safety planning & Free & $\mathrm{N} / \mathrm{A}^{\mathrm{b}}$ & $\begin{array}{ll}\text { - } & \text { Text-based } \\
\text { - } & \text { Workbook or planner }\end{array}$ \\
\hline
\end{tabular}

${ }^{a}$ LGBQ: lesbian, gay, bisexual, and queer.

${ }^{\mathrm{b}} \mathrm{N} / \mathrm{A}$ : not applicable. 
Table 3. Program characteristics of web-based programs with downloadable worksheets or resources.

\begin{tabular}{|c|c|c|c|c|}
\hline Program & $\begin{array}{l}\text { Focus and target population } \\
\text { (if specified) }\end{array}$ & Access & Therapist support & Presentation format \\
\hline \multicolumn{5}{|l|}{ Depression } \\
\hline $\mathrm{CCI}^{\mathrm{a}}$-Depression & Depression & Free & No & - Text workbook with images \\
\hline $\begin{array}{l}\text { Evolution Health-Overcoming } \\
\text { Depression }\end{array}$ & Depression, $\geq 16$ years & Free & Not in free version & $\begin{array}{ll}\text { - } & \text { Text } \\
\text { - } & \text { Gamified quiz }\end{array}$ \\
\hline $\begin{array}{l}\text { Mental health Online-Depres- } \\
\text { sion Online Program }\end{array}$ & Depression, $\geq 18$ years & Free & $\begin{array}{l}\text { Yes, optional access } \\
\text { to e-therapists }\end{array}$ & $\begin{array}{ll}\text { - } & \text { Text-based } \\
\text { - } & \text { Graphics } \\
\text { - } & \text { Audio or video } \\
\text { - } & \text { Characters or case scenarios }\end{array}$ \\
\hline $\begin{array}{l}\text { This Way Up-The Depression } \\
\text { Course }\end{array}$ & Depression, $\geq 18$ years & $\begin{array}{l}\text { Free when referred } \\
\text { by clinician }\end{array}$ & $\begin{array}{l}\text { Yes, independent } \\
\text { clinician allowed ac- } \\
\text { cess }\end{array}$ & $\begin{array}{ll}\text { - } & \text { Text-based } \\
\text { - } & \text { Graphics } \\
\text { - } & \text { Audio or video } \\
\text { - } & \text { Characters or case scenarios } \\
\text { - } & \text { Comic slideshow format }\end{array}$ \\
\hline Students Against Depression & Depression, students & Free & No & - $\quad$ Text-based \\
\hline \multicolumn{5}{|l|}{ Anxiety } \\
\hline $\mathrm{CCI}$-Health and Anxiety & Health anxiety & Free & No & - $\quad$ Text workbook with images \\
\hline $\mathrm{CCI}$-Panic & Panic attacks & Free & No & - Text workbook with images \\
\hline CCI-Social Anxiety & Social anxiety & Free & No & - $\quad$ Text workbook with images \\
\hline $\mathrm{CCI}$-Worry and Rumination & Worry & Free & No & - $\quad$ Text workbook with images \\
\hline $\begin{array}{l}\text { Evolution Health-Overcoming } \\
\text { Anxiety }\end{array}$ & Anxiety, $\geq 16$ years & Free & Not in free version & $\begin{array}{ll}\text { - } & \text { Text } \\
\text { - } & \text { Gamified quiz }\end{array}$ \\
\hline $\begin{array}{l}\text { Evolution Health-Managing } \\
\text { Anxiety Course }\end{array}$ & Anxiety, $\geq 16$ years & Free & Not in free version & $\begin{array}{ll}\text { - } & \text { Text } \\
\text { - } & \text { Gamified quiz }\end{array}$ \\
\hline $\begin{array}{l}\text { Mental Health Online-GAD } \\
\text { program }\end{array}$ & GAD, $\geq 18$ years & Free & $\begin{array}{l}\text { Yes, optional access } \\
\text { to e-therapists }\end{array}$ & $\begin{array}{ll}\text { - } & \text { Text-based } \\
\text { - } & \text { Graphics } \\
\text { - } & \text { Audio or video } \\
\text { - } & \text { Characters or case scenarios }\end{array}$ \\
\hline $\begin{array}{l}\text { Mental Health Online-Panic } \\
\text { STOP! }\end{array}$ & Panic attacks, $\geq 18$ years & Free & $\begin{array}{l}\text { Yes, optional access } \\
\text { to e-therapists }\end{array}$ & $\begin{array}{ll}\text { - } & \text { Text-based } \\
\text { - } & \text { Graphics } \\
\text { - } & \text { Audio or video } \\
\text { - } & \text { Characters or case scenarios }\end{array}$ \\
\hline $\begin{array}{l}\text { Mental Health Online-Social } \\
\text { Anxiety Online Program }\end{array}$ & Social anxiety, $\geq 18$ years & Free & $\begin{array}{l}\text { Yes, optional access } \\
\text { to e-therapists }\end{array}$ & $\begin{array}{ll}\text { - } & \text { Text-based } \\
\text { - } & \text { Graphics } \\
\text { - } & \text { Audio or video } \\
\text { - } & \text { Characters or case scenarios }\end{array}$ \\
\hline $\begin{array}{l}\text { This Way Up_-Health Anxiety } \\
\text { Course }\end{array}$ & Worry about health, $\geq 18$ years & $\begin{array}{l}\text { Free when referred } \\
\text { by clinician }\end{array}$ & $\begin{array}{l}\text { Yes, independent } \\
\text { clinician allowed ac- } \\
\text { cess }\end{array}$ & $\begin{array}{ll}\text { - } & \text { Text-based } \\
\text { - } & \text { Graphics } \\
\text { - } & \text { Audio or video } \\
\text { - } & \text { Characters or case scenarios } \\
\text { - } & \text { Comic slideshow format }\end{array}$ \\
\hline $\begin{array}{l}\text { This Way Up_-Panic Attacks } \\
\text { Course }\end{array}$ & Panic attacks, $\geq 18$ years & $\begin{array}{l}\text { Free when referred } \\
\text { by clinician }\end{array}$ & $\begin{array}{l}\text { Yes, independent } \\
\text { clinician allowed ac- } \\
\text { cess }\end{array}$ & $\begin{array}{ll}\text { - } & \text { Text-based } \\
\text { - } & \text { Graphics } \\
\text { - } & \text { Audio or video } \\
\text { - } & \text { Characters or case scenarios } \\
\text { - } & \text { Comic slideshow format }\end{array}$ \\
\hline
\end{tabular}




\begin{tabular}{|c|c|c|c|c|}
\hline Program & $\begin{array}{l}\text { Focus and target population } \\
\text { (if specified) }\end{array}$ & Access & Therapist support & Presentation format \\
\hline $\begin{array}{l}\text { This Way Up-Social Anxiety } \\
\text { Course }\end{array}$ & Social anxiety, $\geq 18$ years & $\begin{array}{l}\text { Free when referred } \\
\text { by clinician }\end{array}$ & $\begin{array}{l}\text { Yes, independent } \\
\text { clinician allowed ac- } \\
\text { cess }\end{array}$ & $\begin{array}{ll}\text { - } & \text { Text-based } \\
\text { - } & \text { Graphics } \\
\text { - } & \text { Audio or video } \\
\text { - } & \text { Characters or case scenarios } \\
\text { Comic slideshow format }\end{array}$ \\
\hline $\begin{array}{l}\text { This Way Up-Worry Course } \\
\text { (GAD) }\end{array}$ & $\mathrm{GAD}, \geq 18$ years & $\begin{array}{l}\text { Free when referred } \\
\text { by clinician }\end{array}$ & $\begin{array}{l}\text { Yes, independent } \\
\text { clinician allowed ac- } \\
\text { cess }\end{array}$ & $\begin{array}{ll}\text { - } & \text { Text-based } \\
\text { - } & \text { Graphics } \\
\text { - } & \text { Characters or case scenarios } \\
\text { Comic slideshow format }\end{array}$ \\
\hline
\end{tabular}

\section{Multi-issue}

Mental Health Online-Made-4- Can pick up to 3 areas: depres- Free Me Program sion, GAD, panic disorder, PTSD $^{\mathrm{c}}$, social anxiety, $\mathrm{OCD}^{\mathrm{d}}$, $\geq 18$ years

This Way Up-MindfulnessBased CBT ${ }^{\mathrm{e}}$ Course

Depression and anxiety, $\geq 18$ years

Free when referred by clinician

Depression and anxiety, $\geq 18$ years sion and Anxiety Course

This Way Up-MUMentum Pregnancy

Anxiety and low mood, adults $<36$ week pregnant

Free when referred by clinician

Anxiety and low mood, adults $>36$ weeks pregnant or have given birth within the last 12 months

Free when referred by clinician

Free when referred by clinician
Yes, optional access to e-therapists

Yes, independent clinician allowed access

Yes, independent clinician allowed access

- Graphics

- Audio or video

- Characters or case scenarios

- Comic slideshow format

- Text-based

- Graphics

- Audio or video

- Characters or case scenarios

- Comic slideshow format

Yes, independent clinician allowed access

- Text-based

- Graphics

- Characters or case scenarios

- Comic slideshow format

Yes, independent clinician allowed access

- Text-based

- Graphics

- Characters or case scenarios

- Comic slideshow format 


\begin{tabular}{|c|c|c|c|c|}
\hline Program & $\begin{array}{l}\text { Focus and target population } \\
\text { (if specified) }\end{array}$ & Access & Therapist support & Presentation format \\
\hline CCI-Tolerating Distress & Distress & Free & No & - $\quad$ Text workbook with images \\
\hline $\begin{array}{l}\text { Evolution Health—Grief and } \\
\text { Loss }\end{array}$ & Grief, $\geq 16$ years & Free & Not in free version & $\begin{array}{ll}\text { - } & \text { Text } \\
\text { - } & \text { Gamified quiz }\end{array}$ \\
\hline Living Life to the Full for Adults & Emotional well-being, adults & $\begin{array}{l}\text { Free for the basic } \\
\text { course; can upgrade } \\
\text { for a fee }\end{array}$ & No & $\begin{array}{ll}\text { - } & \text { Text-based } \\
\text { - } & \text { Graphics } \\
\text { - } & \text { Audio or video } \\
\text { - } & \text { Comic slideshow format }\end{array}$ \\
\hline $\begin{array}{l}\text { Living Life to the Full for Farm- } \\
\text { ing Communities }\end{array}$ & $\begin{array}{l}\text { Emotional well-being, farm- } \\
\text { ing communities }\end{array}$ & $\begin{array}{l}\text { Free for the basic } \\
\text { course; can upgrade } \\
\text { for a fee }\end{array}$ & No & $\begin{array}{ll}\text { - } & \text { Text-based } \\
\text { - } & \text { Graphics } \\
\text { - } & \text { Audio or video } \\
\text { - } & \text { Comic slideshow format }\end{array}$ \\
\hline Living Life to the Full with God & $\begin{array}{l}\text { Emotional well-being, people } \\
\text { of faith }\end{array}$ & $\begin{array}{l}\text { Free for the basic } \\
\text { course; can upgrade } \\
\text { for a fee }\end{array}$ & No & $\begin{array}{ll}\text { - } & \text { Text-based } \\
\text { - } & \text { Graphics } \\
\text { - } & \text { Audio or video } \\
\text { Comic slideshow format }\end{array}$ \\
\hline
\end{tabular}

${ }^{\mathrm{a}} \mathrm{CCI}$ : Center for Clinical Interventions.

${ }^{\mathrm{b}} \mathrm{GAD}$ : generalized anxiety disorder.

${ }^{\mathrm{c}} \mathrm{PTSD}$ : posttraumatic stress disorder.

${ }^{\mathrm{d} O C D}$ : obsessive compulsive disorder.

${ }^{\mathrm{e}} \mathrm{CBT}$ : cognitive behavioral therapy.

\section{Authorship Details}

The review was limited to programs that were accessible to Australians. Unsurprisingly, most of these programs identified Australia as their country of origin $(40 / 52,77 \%)$, although other countries of origin were Scotland (5/52, 10\%), Canada (4/52, $8 \%$ ), the United States (in collaboration with Australia; 2/52, $4 \%)$ and the United Kingdom (1/52, 2\%). Contact details for the authors or developers were provided by almost all websites $(51 / 52,98 \%)$. An overview of program developers and website links is provided in Multimedia Appendix 2.

\section{Program Characteristics}

Table 2 provides an overview of program characteristics for web-based interactive programs and Table 3 for web-based programs with downloadable worksheets or resources.

\section{Focus and Target Population}

Overall, $17 \%(9 / 52)$ of programs specifically addressed depression, $29 \%$ (15/52) of programs addressed anxiety, $25 \%$ (13/52) of programs addressed multiple issues, $25 \%$ (13/52) of programs addressed general well-being, and $4 \%(2 / 52)$ of programs addressed suicidal ideation. Most programs specifically stated that their target audience was the general adult population (aged $\geq 16$ years or $\geq 18$ years; $26 / 52,50 \%$ ), with other $(9 / 52,17 \%)$ programs not specifying a target group but appearing to be designed for adults, based on content. Several programs were designed for specific target populations, including expectant mothers or new parents $(6 / 52,12 \%)$, tertiary students $(3 / 52,6 \%)$, farmers or rural communities $(2 / 52,4 \%)$,

church-going adults $(1 / 52,2 \%)$, the LGBQ community (1/52, $2 \%)$, Aboriginal and Torres Strait Islander people $(1 / 52,2 \%)$, young adults $(1 / 52,2 \%)$, adults aged 26 to 65 years $(1 / 52,2 \%)$, and older adults $(1 / 52,2 \%)$.

\section{Support and Features}

Approximately $88 \%$ (46/52) of programs provided a statement of professional support, that is, mental health clinicians or researchers were involved in the programs' development. Of the 52 programs, $11(21 \%)$ programs allowed users to invite their independent clinician to monitor their progress in the course, and a further 10 (19\%) programs offered optional therapist support while engaging in the program. Approximately $8 \%(4 / 52)$ of programs (Evolution Health) did not offer therapist support through the free version of their programs; however, therapist support was an optional inclusion for paid versions of the program licensed to organizations. Only 8\% (4/52) of programs (Evolution Health) enabled peer support, such as through forums, and in all cases, this was expert-moderated. Most programs offered multiple forms of information delivery, with text, graphics, audio or video, characters, and case scenarios as common features. Email or SMS text message reminders were available $60 \%(31 / 52)$ of the programs.

\section{Intervention Characteristics}

\section{Overview}

The intervention characteristics of web-based interactive programs and web-based programs with downloadable worksheets or resources are shown in Tables 4 and 5, respectively. 
Table 4. Intervention characteristics of web-based interactive programs.

\begin{tabular}{|c|c|c|c|c|}
\hline Program & $\begin{array}{l}\text { Therapeutic ap- } \\
\text { proach }\end{array}$ & Program structure & Modules (length) & Intervention features \\
\hline \multicolumn{5}{|l|}{ Depression } \\
\hline eCouch—Depression Program & $\mathrm{CBT}^{\mathrm{a}}+\mathrm{IPT}^{\mathrm{b}}$ & Structured & 3 sections & $\begin{array}{ll}\text { - } & \text { Worksheets } \\
\text { - } & \text { Mood or symptom monitoring } \\
\text { - } & \text { Diary }\end{array}$ \\
\hline Mum2B-MoodBooster & CBT & Structured & 6 modules ( 6 weeks) & $\begin{array}{ll}\text { - } & \text { Worksheets } \\
\text { - } & \text { Mood or symptom monitoring }\end{array}$ \\
\hline MumMood-Booster & CBT & Structured & 6 modules ( 6 weeks) & $\begin{array}{ll}\text { - } & \text { Worksheets } \\
\text { - } & \text { Mood or symptom monitoring }\end{array}$ \\
\hline OnTrack-Depression & CBT & Structured & 6 modules ( 8 weeks) & $\begin{array}{ll}\text { - } & \text { Worksheets } \\
\text { - } & \text { Mood or symptom monitoring } \\
\text { - } & \text { Diary } \\
\text { - } & \text { Reminder or calendar feature }\end{array}$ \\
\hline
\end{tabular}

\section{Anxiety}

$\begin{array}{llll}\begin{array}{l}\text { eCouch-Anxiety and Worry Pro- } \\ \text { gram }\end{array} & \text { CBT+IPT } & \text { Structured } & 3 \text { sections } \\ \text { eCouch—Social Anxiety Program } & \text { CBT+IPT } & \text { Unstructured } & 6 \text { sections }\end{array}$

\section{Multi-issue}

MindSpot—Indigenous Wellbeing
MindSpot-Mood Mechanic
MindSpot_Wellbeing
MindSpot-Wellbeing Plus
MoodGym
MyCompass

CBT+ IPT+positive Unstructured psychology

My Digital Health—Life Flex Biopsychosocial ap-
proach+CBT+posi-
tive psychology

My Digital Health—Life Flex $\mathrm{LGBQ}^{\mathrm{d}}$

Biopsychosocial approach $+\mathrm{CBT}+$ positive psychology
- Worksheets

- Mood or symptom monitoring

- Diary

- Worksheets

$\begin{array}{ll}\text { Structured } & 5 \text { modules (8 weeks) } \\ \text { Structured } & 5 \text { modules (8 weeks) } \\ \text { Structured } & 5 \text { modules ( } 8 \text { weeks) } \\ \text { Structured } & 5 \text { modules (8 weeks) } \\ \text { Structured } & 5 \text { modules }\end{array}$

Structured (optional 7 modules (scheduled reor mandatory depending on $\mathrm{RCT}^{\mathrm{c}}$ arm)

\section{RCT arm)}

14 activities (recommend 7 weeks)
- Worksheets

- Worksheets

- Worksheets

- Worksheets

- Worksheets

- Mood or symptom monitoring

Worksheets

- Mood or symptom monitoring

- Diary

- Worksheets

- Mood or symptom monitoring

- Diary

- Option to connect Fitbit or other health monitoring tools

- Safety planning tool

Structured (optional 7 modules (scheduled reor mandatory de- lease over 7 weeks in 1 pending on RCT RCT arm) arm)

- Worksheets

- Mood or symptom monitoring

- Diary

- Option to connect Fitbit or other health monitoring tools

- Safety planning tool

\section{General well-being}

eCouch-Bereavement and Loss CBT Program
Structured

2 sections
- Worksheets

- Mood or symptom monitoring

- Diary 


\begin{tabular}{|c|c|c|c|c|}
\hline Program & $\begin{array}{l}\text { Therapeutic ap- } \\
\text { proach }\end{array}$ & Program structure & Modules (length) & Intervention features \\
\hline eCouch-Divorce and Separation & CBT & Structured & 3 sections & $\begin{array}{ll}- & \text { Worksheets } \\
\text { - } & \text { Mood or symptom monitoring } \\
\text { - } & \text { Diary }\end{array}$ \\
\hline ifarmwell & $\mathrm{ACT}^{\mathrm{e}}$ & Structured & 5 modules ( 10 weeks) & $\begin{array}{ll}\text { - } & \text { Worksheets } \\
\text { - } & \text { Personalized script }\end{array}$ \\
\hline The Desk & $\begin{array}{l}\text { CBT+mindful- } \\
\text { ness+positive psy- } \\
\text { chology }\end{array}$ & Unstructured & 4 modules & $\begin{array}{l}\text { - } \quad \text { Mood or symptom monitoring } \\
\text { - } \quad \text { Reminder or calendar feature }\end{array}$ \\
\hline \multicolumn{5}{|l|}{ Suicidal ideation } \\
\hline My Digital Health—iConsiderLife & No & Structured & 6 pathways & - Worksheets \\
\hline BeyondNow & $N / A^{f}$ & Unstructured & No modules & $\begin{array}{l}\text { - Process of completing plan is } \\
\text { like a worksheet }\end{array}$ \\
\hline
\end{tabular}

${ }^{\mathrm{a} C B T}$ : cognitive behavioral therapy.

${ }^{b}$ IPT: interpersonal psychotherapy.

${ }^{\mathrm{c}} \mathrm{RCT}$ : randomized controlled trial.

${ }^{\mathrm{d}}$ LGBQ: lesbian, gay, bisexual, and queer.

${ }^{\mathrm{e}} \mathrm{ACT}$ : acceptance and commitment therapy.

${ }_{\mathrm{f}} \mathrm{N} / \mathrm{A}$ : not applicable. 
Table 5. Intervention characteristics of web-based programs with downloadable worksheets or resources.

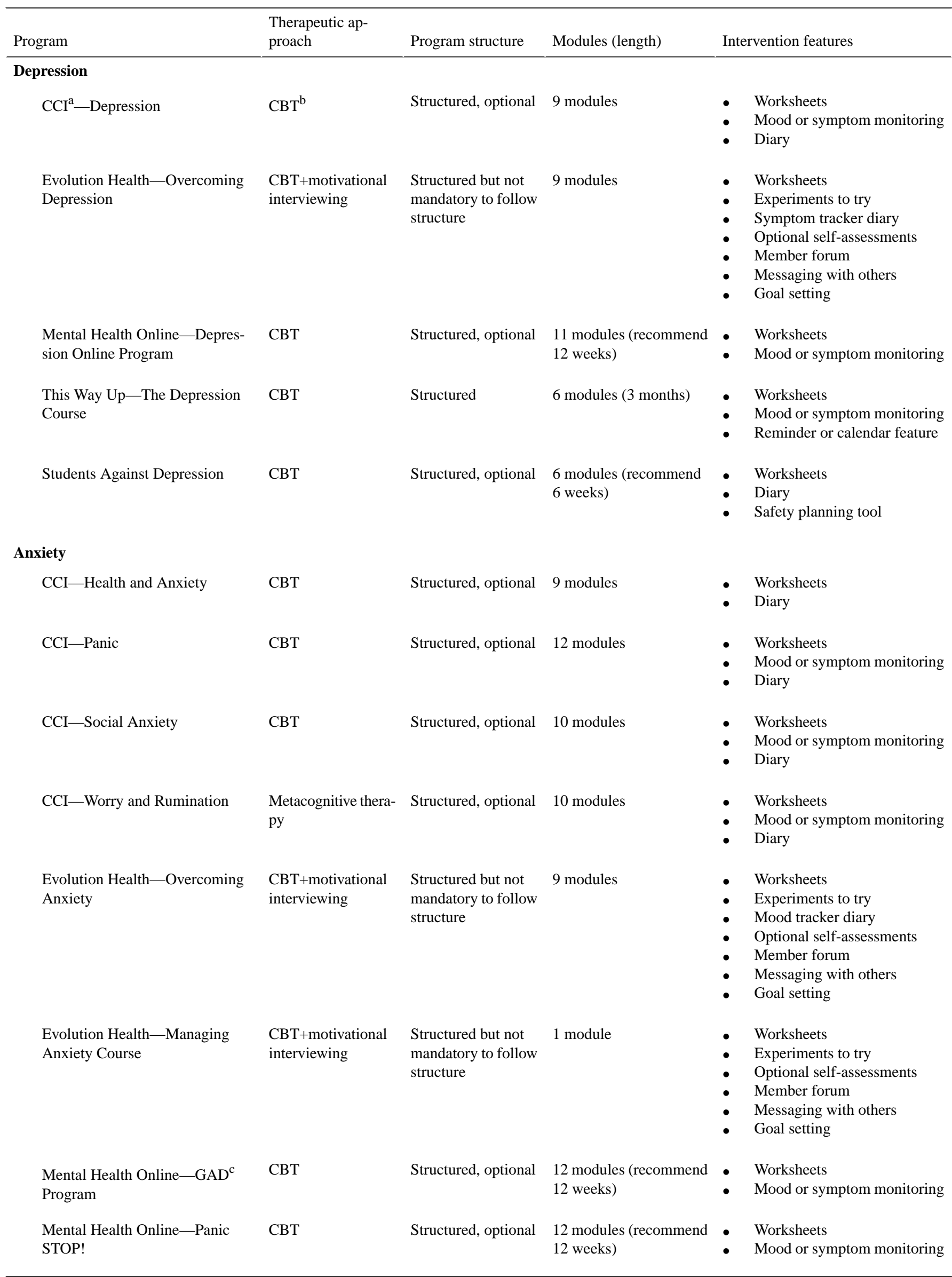




\begin{tabular}{|c|c|c|c|c|}
\hline Program & $\begin{array}{l}\text { Therapeutic ap- } \\
\text { proach }\end{array}$ & Program structure & Modules (length) & Intervention features \\
\hline $\begin{array}{l}\text { Mental Health Online-_Social } \\
\text { Anxiety Online Program }\end{array}$ & CBT & Structured, optional & $\begin{array}{l}12 \text { modules (recommend } \\
12 \text { weeks) }\end{array}$ & $\begin{array}{ll}\text { - } & \text { Worksheets } \\
\text { - } & \text { Mood or symptom monitoring }\end{array}$ \\
\hline $\begin{array}{l}\text { This Way Up-Health Anxiety } \\
\text { Course }\end{array}$ & CBT & Structured & 6 modules ( 3 months) & $\begin{array}{ll}\text { - } & \text { Worksheets } \\
\text { - } & \text { Mood or symptom monitoring } \\
\text { - } & \text { Reminder or calendar feature }\end{array}$ \\
\hline $\begin{array}{l}\text { This Way Up_-Panic Attacks } \\
\text { Course }\end{array}$ & CBT & Structured & 6 modules ( 3 months) & $\begin{array}{ll}\text { - } & \text { Worksheets } \\
\text { - } & \text { Mood or symptom monitoring } \\
\text { - } & \text { Reminder or calendar feature }\end{array}$ \\
\hline $\begin{array}{l}\text { This Way Up-Social Anxiety } \\
\text { Course }\end{array}$ & CBT & Structured & 6 modules (3 months) & $\begin{array}{ll}\text { - } & \text { Worksheets } \\
\text { - } & \text { Mood or symptom monitoring } \\
\text { - } & \text { Reminder or calendar feature }\end{array}$ \\
\hline $\begin{array}{l}\text { This Way Up_-Worry Course } \\
\text { (GAD) }\end{array}$ & CBT & Structured & 6 modules (3 months) & $\begin{array}{ll}\text { - } & \text { Worksheets } \\
\text { - } & \text { Mood or symptom monitoring } \\
\text { - } & \text { Reminder or calendar feature }\end{array}$ \\
\hline \multicolumn{5}{|l|}{ Multi-issue } \\
\hline $\begin{array}{l}\text { Mental Health Online-_Made-4- } \\
\text { Me Program }\end{array}$ & CBT & Structured, optional & $\begin{array}{l}11 \text { modules (recommend } \\
12 \text { weeks) }\end{array}$ & $\begin{array}{ll}\text { - } & \text { Worksheets } \\
\text { - } & \text { Mood or symptom monitoring }\end{array}$ \\
\hline $\begin{array}{l}\text { This Way Up-Mindfulness-based } \\
\text { CBT Course }\end{array}$ & $\mathrm{CBT}+$ mindfulness & Structured & 6 modules (3 months) & $\begin{array}{ll}\text { - } & \text { Worksheets } \\
\text { - } & \text { Mood or symptom monitoring } \\
\text { - } & \text { Reminder or calendar feature }\end{array}$ \\
\hline $\begin{array}{l}\text { This Way Up-Mixed Depression } \\
\text { and Anxiety Course }\end{array}$ & CBT & Structured & 6 modules (3 months) & $\begin{array}{ll}\text { - } & \text { Worksheets } \\
\text { - } & \text { Mood or symptom monitoring } \\
\text { - } & \text { Reminder or calendar feature }\end{array}$ \\
\hline $\begin{array}{l}\text { This Way Up-MUMentum Preg- } \\
\text { nancy }\end{array}$ & CBT & Structured & 3 modules (4-6 weeks) & $\begin{array}{ll}\text { - } & \text { Worksheets } \\
\text { - } & \text { Mood or symptom monitoring } \\
\text { - } & \text { Reminder or calendar feature }\end{array}$ \\
\hline $\begin{array}{l}\text { This Way Up-MUMentum Post- } \\
\text { natal }\end{array}$ & CBT & Structured & 3 modules (4-6 weeks) & $\begin{array}{ll}\text { - } & \text { Worksheets } \\
\text { - } & \text { Mood or symptom monitoring } \\
\text { - } & \text { Reminder or calendar feature }\end{array}$ \\
\hline General well-being & & & & \\
\hline
\end{tabular}




\begin{tabular}{|c|c|c|c|c|}
\hline Program & $\begin{array}{l}\text { Therapeutic ap- } \\
\text { proach }\end{array}$ & Program structure & Modules (length) & Intervention features \\
\hline CCI-Tolerating Distress & $\mathrm{DBT}^{\mathrm{d}}$ & Structured, optional & 4 modules & $\begin{array}{ll}\text { - } & \text { Worksheets } \\
\text { - } & \text { Mood or symptom monitoring } \\
\text { - } & \text { Diary }\end{array}$ \\
\hline Evolution Health - Grief and Loss & $\begin{array}{l}\text { CBT+motivational } \\
\text { interviewing }\end{array}$ & $\begin{array}{l}\text { Structured but not } \\
\text { mandatory to follow } \\
\text { structure }\end{array}$ & 1 module & $\begin{array}{ll}\text { - } & \text { Worksheets } \\
\text { - } & \text { Experiments to try } \\
\text { - } & \text { Optional self-assessments } \\
\text { - } & \text { Member forum } \\
\text { - } & \text { Messaging with others } \\
\text { - } & \text { Goal setting }\end{array}$ \\
\hline Living Life to the Full for Adults & CBT & Structured, optional & $\begin{array}{l}8 \text { modules (recommend } \\
8 \text { weeks) }\end{array}$ & $\begin{array}{ll}\text { - } & \text { Worksheets } \\
\text { - } & \text { Mood or symptom monitoring } \\
\text { - } & \text { Blog } \\
\text { - } & \text { Reminder or calendar feature }\end{array}$ \\
\hline $\begin{array}{l}\text { Living Life to the Full for Farming } \\
\text { Communities }\end{array}$ & CBT & Structured, optional & 5 modules & $\begin{array}{ll}\text { - } & \text { Worksheets } \\
\text { - } & \text { Mood or symptom monitoring } \\
\text { - } & \text { Diary } \\
\text { - } & \text { Blog } \\
\text { - } & \text { Reminder or calendar feature }\end{array}$ \\
\hline Living Life to the Full with God & CBT & Structured, optional & $\begin{array}{l}8 \text { modules (recommend } \\
8 \text { weeks) }\end{array}$ & $\begin{array}{ll}\text { - } & \text { Worksheets } \\
\text { - } & \text { Mood or symptom monitoring } \\
\text { - } & \text { Diary } \\
\text { - } & \text { Blog } \\
\text { - } & \text { Reminder or calendar feature }\end{array}$ \\
\hline $\begin{array}{l}\text { Living Life to the Full _-Enjoy } \\
\text { Your Baby }\end{array}$ & CBT & Structured, optional & 5 modules & $\begin{array}{ll}\text { - } & \text { Worksheets } \\
\text { - } & \text { Blog } \\
\text { - } & \text { Reminder or calendar feature }\end{array}$ \\
\hline $\begin{array}{l}\text { Living Life to the Full _-Enjoy } \\
\text { Your Bump }\end{array}$ & CBT & Structured, optional & 5 modules & $\begin{array}{ll}\text { - } & \text { Worksheets } \\
\text { - } & \text { Mood or symptom monitoring } \\
\text { - } & \text { Blog } \\
\text { - } & \text { Reminder or calendar feature }\end{array}$ \\
\hline $\begin{array}{l}\text { This Way Up-Coping with Stress } \\
\text { Course }\end{array}$ & CBT & Structured & 4 modules ( 3 months) & $\begin{array}{ll}\text { - } & \text { Worksheets } \\
\text { - } & \text { Mood or symptom monitoring } \\
\text { - } & \text { Reminder feature } \\
\text { - } & \text { Calendar feature }\end{array}$ \\
\hline $\begin{array}{l}\text { This Way Up-The Student Well- } \\
\text { being Course }\end{array}$ & CBT & Structured & 8 modules ( 3 months) & $\begin{array}{ll}\text { - } & \text { Worksheets } \\
\text { - } & \text { Mood or symptom monitoring } \\
\text { - } & \text { Reminder or calendar feature }\end{array}$ \\
\hline
\end{tabular}

${ }^{\mathrm{a} C C I}$ : Centre for Clinical Interventions.

${ }^{\mathrm{b}} \mathrm{CBT}$ : cognitive behavioral therapy.

${ }^{\mathrm{c}} \mathrm{GAD}$ : generalized anxiety disorder.

${ }^{\mathrm{d}}$ DBT: dialectical behavior therapy.

\section{Therapeutic Approach or Model of Change}

On the basis of either self-descriptions or the clinical judgment of the authors, $90 \%$ (47/52) of programs were based on cognitive behavioral therapy (CBT), either with $21 \%$ (11/52) or without $69 \%(36 / 52)$ other therapeutic approaches. In combination with CBT, some programs included motivational interviewing $(4 / 52$, $8 \%)$, interpersonal therapy $(4 / 52,8 \%)$, or mindfulness $(1 / 52$, $2 \%)$. Only $2 \%(1 / 52)$ of programs (ifarmwell) was based on acceptance and commitment therapy (ACT), 2\% (1/52) of programs (Centre for Clinical Interventions [CCI]: Tolerating
Distress) on dialectical behavior therapy (DBT), and 2\% (1/52) of programs (BeyondNow) on problem solving (ie, how to keep self safe or safety planning).

\section{Type and Dose of Intervention}

In total, $48 \%$ (25/52) of programs were structured or tunneled, where participants were led through a series of modules or sessions in a specific order. A further $42 \%$ (22/52) of programs recommended to follow the program in a specific order but allowed users to choose the order in which they completed them. Approximately $4 \%(2 / 52)$ of programs were part of an RCT 
research project that had open access and allocated participants to 1 of 2 therapy structure conditions: mandatory (participants did not choose the order) or optional (participants were given the freedom to choose the order of completion). Approximately $6 \%(3 / 52)$ of programs were unstructured.

The number of modules or activities offered within a program ranged from 1 to 14 (mean 6.51, SD 3.03), except for 1 program (BeyondNow), which offered a safety planner instead of modules. The recommended time to complete the modules ranged from 1 week to 3 months (specified for $34 / 52$, 65\% of programs). Approximately 33\% (17/52) of programs did not recommend a set treatment length. Self-assessments were included in $79 \%(41 / 52)$ programs, and it was mandatory in $56 \%(29 / 52)$ of programs and optional in $23 \%(12 / 52)$ of programs.

\section{Intervention Features}

All programs, except 4\% (2/52; The Desk and iConsider Life), featured worksheets, either within the web-based intervention or as downloadable forms to be completed offline. Diaries were featured in $46 \%(24 / 52)$ of programs, and $75 \%$ (39/52) of programs included some type of mood or symptom monitoring.

\section{Empirical Evidence: Program Evaluation}

Out of 17 possible evaluation items, 75\% (39/52) of programs could be evaluated against all 17 items, $15 \%$ (8/52) against $94 \%$ $(16 / 17)$ of items, and $10 \%(5 / 52)$ against $76 \%(13 / 17)$ of items (Table 6). In total, scores ranged from $44 \%$ to $100 \%$, with an average score of $85 \%$ (SD 10.7\%). More specifically, of the $75 \%(39 / 52)$ of programs that provided values for all 17 items, scores varied between $65 \%$ and $94 \%$, with an average score of $87 \%$ (SD 7\%). This was between $44 \%$ and $81 \%$, with an average of $69 \%$ (SD 11\%), for programs that provided values for 16 items, and between $85 \%$ and $100 \%$, with an average of $95 \%$ (SD 7\%), for programs that provided values for 13 items. Individual answers to the program evaluation criteria are shown in Multimedia Appendix 3. 
Table 6. Type of research conducted to evaluate eligible programs, including the level of evidence and process evaluation scores ( $\mathrm{N}=52$ ).

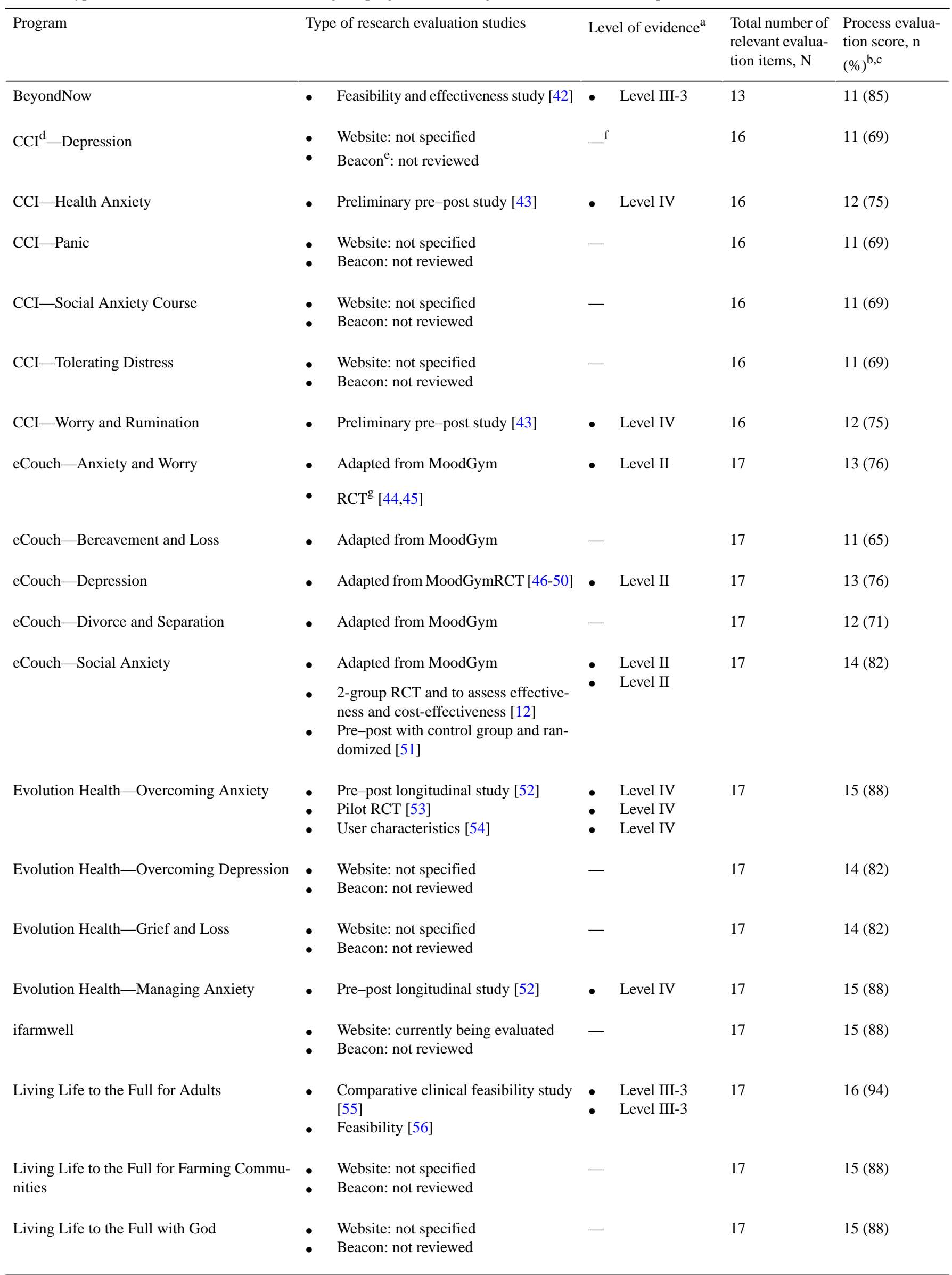




\begin{tabular}{|c|c|c|c|c|}
\hline Program & Type of research evaluation studies & Level of evidence ${ }^{\mathrm{a}}$ & $\begin{array}{l}\text { Total number of } \\
\text { relevant evalua- } \\
\text { tion items, } \mathrm{N}\end{array}$ & $\begin{array}{l}\text { Process evalua- } \\
\text { tion score, } \mathrm{n} \\
(\%)^{\mathrm{b}, \mathrm{c}}\end{array}$ \\
\hline Living Life to the Full—Enjoy Your Baby & $\begin{array}{l}\text { - Website: not specified } \\
\text { - } \quad \text { Beacon: not reviewed }\end{array}$ & - & 17 & $15(88)$ \\
\hline Living Life to the Full—Enjoy Your Bump & $\begin{array}{l}\text { - Website: not specified } \\
\text { - } \quad \text { Beacon: not reviewed }\end{array}$ & - & 17 & $15(88)$ \\
\hline Mental Health Online-Depression Online & $\begin{array}{l}\text { - Uncontrolled pre-post treatment study } \\
{[57,58]}\end{array}$ & - $\quad$ Level IV & 17 & $15(88)$ \\
\hline $\begin{array}{l}\text { Mental Health Online-Generalized Anxiety } \\
\text { Disorder }\end{array}$ & $\begin{array}{l}\text { - Uncontrolled pre-post treatment study } \\
\text { [57] } \\
\text { - } \quad \text { Pre-post treatment [59] } \\
\text { - } \quad \text { Pre- to posttreatment quasi-experimen- } \\
\text { tal (participant choice) [60] }\end{array}$ & $\begin{array}{ll}\text { - } & \text { Level IV } \\
\text { - } & \text { Level IV } \\
\text { - } & \text { Level IV }\end{array}$ & 17 & $15(88)$ \\
\hline Mental Health Online-Made $4 \mathrm{Me}$ & $\begin{array}{l}\text { - Website: not specified } \\
\text { - } \quad \text { Beacon: not reviewed }\end{array}$ & - & 17 & $15(88)$ \\
\hline Mental Health Online_-Panic STOP! & $\begin{array}{ll}\text { - } & \text { Uncontrolled pre-post treatment study } \\
& \text { [57] } \\
\text { - } & \text { Pre-post treatment [59] } \\
\text { - } & \text { Participant choice quasi-experimental } \\
& \text { trial [60] }\end{array}$ & $\begin{array}{ll}\text { - } & \text { Level IV } \\
\text { - } & \text { Level IV } \\
\text { - } & \text { Level IV }\end{array}$ & 17 & $15(88)$ \\
\hline $\begin{array}{l}\text { Mental Health Online-Social Anxiety On- } \\
\text { line }\end{array}$ & $\begin{array}{l}\text { - Pre-post treatment study [59] } \\
\text { - Participant choice quasi-experimental } \\
\text { trial [60] }\end{array}$ & $\begin{array}{ll}- & \text { Level IV } \\
- & \text { Level IV }\end{array}$ & 17 & $15(88)$ \\
\hline MindSpot-Indigenous Wellbeing Course & $\begin{array}{l}\text { - Prospective uncontrolled observational } \\
\text { cohort study [61] }\end{array}$ & - $\quad$ Level IV & 13 & $13(100)$ \\
\hline MindSpot—Mood Mechanic Course & $\begin{array}{ll}\text { - } & \text { Single-arm, open trial [62] } \\
\text { - } & \text { RCT [63] }\end{array}$ & $\begin{array}{ll}\text { - } & \text { Level III-3 } \\
\text { - } & \text { Level II }\end{array}$ & 13 & $13(100)$ \\
\hline MindSpot—Wellbeing & $\begin{array}{ll}\text { - } & \text { Cost-effectiveness [64] } \\
\text { - } & \text { Feasibility trial [65] } \\
\text { - } & \text { RCT [66-73] } \\
\text { - } & \text { 12-month follow-up RCT [62] } \\
\text { - } & \text { Single group open trial [74] }\end{array}$ & $\begin{array}{ll}\text { - } & \text { Level III-3 } \\
\text { - } & \text { Level II } \\
\text { - } & \text { Level II } \\
\text { - } & \text { Level III-3 }\end{array}$ & 13 & $13(100)$ \\
\hline MindSpot—Wellbeing Plus & $\begin{array}{ll}- & \text { Cost-effectiveness }[75,76] \\
- & \text { Feasibility study }[75,77] \\
- & \text { Implementation }[78] \\
- & \text { RCT }[75,76,79,80]\end{array}$ & $\begin{array}{ll}\text { - } & \text { Level II } \\
\text { - } & \text { Level II and } \\
& \text { III-3 } \\
\text { - } & \text { Level II } \\
\text { - } & \text { Level II }\end{array}$ & 13 & $13(100)$ \\
\hline MoodGym & $\begin{array}{ll}\text { - } & \text { Acceptability study [81] } \\
\text { - } & \text { Implementation [82] } \\
\text { - } & \text { Follow-up outcome analysis [83] } \\
\text { - } & \text { Program use [84] } \\
\text { - } & \text { School or class trials }[100,101] \\
\text { - } & \text { Compliance [102] }\end{array}$ & $\begin{array}{ll}\text { - } & \text { Level II } \\
\text { - } & \text { Level II } \\
\text { - } & \text { Level II } \\
\text { - } & \text { Level II } \\
\text { - } & \text { Level III-2 } \\
\text { - } & \text { Level II }\end{array}$ & 17 & $15(88)$ \\
\hline Mum2BMoodBooster & $\begin{array}{l}\text { - Website: currently being evaluated } \\
\text { - Beacon: not reviewed }\end{array}$ & - & 17 & $14(82)$ \\
\hline MumMoodBooster & $\begin{array}{ll}\text { - } & \text { Acceptability study }[103,104] \\
\text { - } & \text { Feasibility study [104] } \\
\text { - } & \text { RCT [105] }\end{array}$ & $\begin{array}{ll}\text { - } & \text { Level III-3 } \\
\text { - } & \text { Level III-3 } \\
\text { - } & \text { Level II }\end{array}$ & 17 & $15(88)$ \\
\hline myCompass & & & 17 & $16(94)$ \\
\hline
\end{tabular}




\begin{tabular}{|c|c|c|c|c|}
\hline Program & Type of research evaluation studies & Level of evidence $^{\mathrm{a}}$ & $\begin{array}{l}\text { Total number of } \\
\text { relevant evalua- } \\
\text { tion items, } \mathrm{N}\end{array}$ & $\begin{array}{l}\text { Process evalua- } \\
\text { tion score, } \mathrm{n} \\
(\%)^{\mathrm{b}, \mathrm{c}}\end{array}$ \\
\hline & $\begin{array}{ll}- & \text { Feasibility study [106] } \\
\text { - } & \text { Open trial [107] } \\
\text { - } & \text { RCT [108-110] }\end{array}$ & $\begin{array}{ll}\text { - } & \text { Level II-3 } \\
\text { - } & \text { Level III-3 } \\
\text { - } & \text { Level II }\end{array}$ & & \\
\hline My Digital Health—iConsiderLife & $\begin{array}{l}\text { - Website: currently being evaluated } \\
\text { - Beacon: not reviewed }\end{array}$ & - & 16 & $13(81)$ \\
\hline My Digital Health—Life Flex & $\begin{array}{l}\text { - Website: currently being evaluated } \\
\text { - Beacon: not reviewed }\end{array}$ & - & 17 & $15(88)$ \\
\hline My Digital Health—Life Flex LGBQ ${ }^{\text {h }}$ & $\begin{array}{l}\text { - Website: currently being evaluated } \\
\text { - Beacon: not reviewed }\end{array}$ & - & 17 & $15(88)$ \\
\hline OnTrack-Depression & $\begin{array}{l}\text { - Website: not specified } \\
\text { - } \quad \text { Beacon: not reviewed }\end{array}$ & - & 17 & $13(76)$ \\
\hline The Desk & $\begin{array}{l}\text { - Website: not specified } \\
\text { - } \quad \text { Beacon: not reviewed }\end{array}$ & - & 17 & $14(82)$ \\
\hline This Way Up—Coping with Stress Course & $\begin{array}{l}\text { - Website: not specified } \\
\text { - } \quad \text { Beacon: not reviewed }\end{array}$ & - & 17 & $15(88)$ \\
\hline This Way Up-The Depression Course & $\begin{array}{ll}\text { - } & \text { Nonrandomized comparison study } \\
& {[111]} \\
\text { - } & \text { Open trial }[112-117] \\
\text { - } & \text { RCT }[111,118-124] \\
\text { - } & \text { Adherence }[125]\end{array}$ & $\begin{array}{ll}\text { - } & \text { Level III-2 } \\
\text { - } & \text { Level III-3 } \\
\text { - } & \text { Level II-3 } \\
\text { - } & \text { Level III-3 }\end{array}$ & 17 & $16(94)$ \\
\hline This Way Up—Health Anxiety Course & $\begin{array}{l}\text { - Open trial }[126,127] \\
\text { - } \quad \text { RCT }[128]\end{array}$ & $\begin{array}{ll}\text { - } & \text { Level III-3 } \\
\text { - } & \text { Level II }\end{array}$ & 17 & $16(94)$ \\
\hline This Way UpMindfulness-based CBT ${ }^{\mathrm{i}}$ Course & $\begin{array}{ll}- & \text { Open trial [129] } \\
- & \text { RCT [130] }\end{array}$ & $\begin{array}{ll}- & \text { Level III-3 } \\
- & \text { Level II }\end{array}$ & 17 & $16(94)$ \\
\hline $\begin{array}{l}\text { This Way Up-Mixed Depression and Anxi- } \\
\text { ety Course }\end{array}$ & $\begin{array}{l}\text { - } \quad \text { Open trial }[74,111,131-133] \\
\text { - } \\
\text { RCT }[72,131,134]\end{array}$ & $\begin{array}{ll}\text { - } & \text { Level III-3 } \\
\text { - } & \text { Level II }\end{array}$ & 17 & $16(94)$ \\
\hline This Way Up-MUMentum Pregnancy & - $\quad$ RCT [135] & - Level II & 17 & $16(94)$ \\
\hline This Way Up—MUMentum Postnatal & - $\quad \mathrm{RCT}[136]$ & - $\quad$ Level II & 17 & $16(94)$ \\
\hline This Way Up_-Panic Attacks Course & $\begin{array}{ll}\text { - } & \text { Open trial [137] } \\
\text { - } & \text { RCT [71,72,138-140] }\end{array}$ & $\begin{array}{ll}\text { - } & \text { Level III-3 } \\
\text { - } & \text { Level II }\end{array}$ & 17 & $16(94)$ \\
\hline This Way Up—Social Anxiety Course & $\begin{array}{ll}\text { - } & \text { Open trial }[141] \\
\text { - } & \text { RCT }[71,72,138,142-146]\end{array}$ & $\begin{array}{ll}\text { - } & \text { Level III-3 } \\
\text { - } & \text { Level II }\end{array}$ & 17 & $16(94)$ \\
\hline This Way Up—Student Wellbeing Course & $\begin{array}{l}\text { - Website: not specified } \\
\text { - Beacon: not reviewed }\end{array}$ & - & 17 & $15(88)$ \\
\hline This Way Up—Worry Course $\left(G^{\prime} D^{j}\right)$ & $\begin{array}{ll}\text { - } & \text { Nonrandomized comparison study } \\
& {[111]} \\
\text { - } & \text { Open trial }[112,133,147,148] \\
\text { - } & \text { RCT }[71,72,138,149-151]\end{array}$ & $\begin{array}{ll}\text { - } & \text { Level III-2 } \\
\text { - } & \text { Level III-3 } \\
\text { - } & \text { Level II }\end{array}$ & 17 & $16(94)$ \\
\hline Students Against Depression & $\begin{array}{l}\text { - Website: currently being evaluated } \\
\text { - Beacon: not reviewed }\end{array}$ & - & 16 & $7(44)$ \\
\hline
\end{tabular}

${ }^{a}$ Level of evidence determined from the National Health and Medical Research Council evidence hierarchy by intervention studies [41]; level I: systematic review; level II: randomized controlled trials; level III-1: pseudorandomized controlled trial; level III-2: comparative study with controls; level III-3: comparative study without controls; and level IV: case series. 
${ }^{\mathrm{b}}$ A program evaluation scoring system was adapted from previously published guidelines for evaluating and reporting web-based intervention research [40].

${ }^{\mathrm{c}}$ Owing to access restrictions, not all programs could be evaluated against all 17 items, which may have resulted in higher scores.

${ }^{\mathrm{d}}$ CCI: Centre for Clinical Interventions.

${ }^{\mathrm{e}}$ Beacon is an Australian clinical web-based platform that describes different web-based self-help treatment programs.

${ }^{\mathrm{f}}$ Evaluation studies are not available.

$\mathrm{g}_{\mathrm{RCT}}$ : randomized controlled trial.

${ }^{\mathrm{h}}$ LGBQ: lesbian, gay, bisexual, and queer.

${ }^{\mathrm{i}} \mathrm{CBT}$ : cognitive behavioral therapy.

${ }^{\mathrm{j}} \mathrm{GAD}$ : generalized anxiety disorder.

Evidence of the program's usability (ie, attrition data, success rate, completion rate, or number of users in the program or testimonials) was provided for $52 \%$ (27/52) of programs. In many cases, where a website offered $\geq 1$ program, these results were provided for the suite of programs listed on the website generally rather than for the specific programs (eg, Evolution Health and This Way Up). Approximately 56\% (29/52) of programs were supported by some type of evaluation; $59 \%$ $(17 / 29)$ had undergone effectiveness trials, and $41 \%(12 / 29)$ had empirical evidence of both effectiveness and acceptability (ie, level III and level IV evidence). Approximately 68\% (19/29) of the evaluated programs had undergone evaluation via RCT (ie, level II evidence). Of the 23 programs without evaluation data, $6(26 \%)$ are currently in the process of being evaluated. All 52 programs and the type of evaluation studies conducted, including the level of evidence, are shown in Table 6 [41]. A rapid review of the evidence for each of these programs' evaluations is provided in Multimedia Appendix 4 [10,12,17,42-151].

\section{Legal and Ethical Issues}

The vast majority of programs provided privacy notices (50/52, $96 \%$ ) and terms and conditions $(51 / 52,98 \%)$. Approximately $88 \%(46 / 52)$ of programs provided crisis links in the form of telephone numbers for emergency services, numbers of helplines or links to websites, and other resources. Only 1 website did not offer crisis links for its programs (CCI); this website was developed by the Western Australian Department of Health to provide evidence-based web-based resources to support practitioners, as well as to provide self-help materials for individuals. The terms and conditions of the website state that the information is provided for information purposes only.

\section{Discussion}

\section{Principal Findings}

The purpose of this scoping review was to summarize the freely available web-based resources based on an evidence-based therapeutic approach for Australian adults seeking self-help for depression, anxiety, suicidal ideation, or general well-being. We sought to describe their characteristics, including therapeutic approaches, key features, and the quality of evidence behind them and thereby produce an accessible summary to inform clinicians' selection of web-based interventions for their patients. This review builds on past reviews of web-based resources for depression [15] and anxiety [16]. To our knowledge, this is the first review of publicly available interventions that includes both suicidal ideation and general well-being.

A total of 52 web-based programs were identified, of which 20 $(39 \%)$ were classified as web-based interactive programs, and $32(62 \%)$ were classified as web-based programs with downloadable worksheets or resources. Of these 52 programs, $29(56 \%)$ had been empirically evaluated, and most of the evidence was assessed as level II or III studies. This is similar to past reviews, which found that $50 \%$ of the programs addressing anxiety had undergone empirical evaluation [16], and $38 \%$ of programs addressing depression had been evaluated via an RCT (ie, level II studies) [15]. A total of 6 additional programs in this review were also currently undergoing evaluation at the time of writing (ifarmwell, My Digital Health programs, Mum2BMoodBooster, and Students Against Depression).

The evaluation of the included programs showed that, on average, programs scored $85 \%$, indicating that the included web-based programs are generally of good quality. The lowest score was for Students Against Depression (44\%) as it did not include information about the developers or the evidence behind the program, privacy notices, terms and conditions. However, Students Against Depression was one of the programs that offered downloadable modules, similar to the CCI programs, and it is currently undergoing evaluation, which may lead to a higher evaluation score once completed. The CCI programs, which scored third to last but are highly regarded by clinicians, included a privacy notice and terms and conditions, and their scores ranged between $69 \%$ and $75 \%$. The highest scores were given to the 4 programs by MindSpot, which could only be evaluated against $77 \%(13 / 17)$ of the items. These programs could not be evaluated on more of the items because of restrictions on accessing the program, which may have resulted in higher scores.

Almost all programs reported that they were based on CBT, either with or without other therapeutic approaches. Only 1 program, ifarmwell, was based on ACT. Although traditional CBT focuses on challenging unhelpful thoughts, ACT focuses on defusing from and accepting them and finding value-consistent ways of living despite the circumstances to build psychological flexibility [152]. A recent international systematic review and meta-analysis of internet-based ACT programs between 2009 and 2019 [153] found 25 efficacy studies in a variety of clinical and nonclinical populations. Overall, the pooled results showed a reduction in symptoms of depression and anxiety and an improvement in quality of life 
and psychological flexibility at postassessment, with results maintained at follow-up assessments. Given this evidence, it is surprising that not more ACT-based web- interventions are available in Australia. However, that review focused on research-driven studies and not publicly available programs; therefore, none of the studies examined by Thompson et al [153] overlapped with this review. Similarly, only 1 program was based on DBT, which differs from CBT in that its main focus is on helping people change their behavior patterns instead of changing dysfunctional beliefs [154,155]. It also focuses on distress tolerance, which is more like ACT than CBT, and includes mindfulness and interpersonal skills training. A review of clinical effectiveness and guidelines showed that DBT is not statistically significantly greater than comparators in reducing depressive and anxiety symptoms [156].

The results of the current review found that $19 \%(10 / 52)$ of programs provided free therapist support, and 21\% (11/52) allowed users to link in their existing clinicians. Research examining whether therapist-guided versus self-guided web-based interventions are more, less, or equally effective has produced mixed results. A recent meta-analysis of 21 studies of web-based programs targeting depression found no significant differences in the effectiveness of the programs to prevent depression based on whether they were guided or not [31]. In contrast, another meta-analysis of studies reported greater effect sizes for outcomes from interventions for depression and anxiety that were guided compared with effect sizes for those that were not [153]. However, these differences may be because of the type of therapy given, as the former was based on CBT and the latter on ACT. In addition, the use of email or SMS text message reminders or messages of encouragement may compensate for the lack of guidance in some programs. Guided interventions are not always practical to implement and may be expensive and hard to sustain and limit the accessibility of the program. Therefore, future research should continue to examine the impact of incorporating, or not incorporating, these program components and alternatives.

This review found that roughly half of the programs were structured or tunneled; a further 22 programs provided a recommended structure (but did not require that the user follow this), and 3 programs were unstructured, allowing the user choice in how and when they completed different modules. Research evidence is inconclusive on the manner in which web-based materials are best organized [157]. A recent systematic review found inconclusive evidence linking website structure and behavioral or health outcomes [158]. They reported that the number of peer-reviewed studies that manipulated website structure to examine its effects on outcome measures was too few to enable conclusive comments to be made. Other studies have provided evidence that allowing unstructured use of websites may increase engagement with the website; however, this may or may not translate into behavior change [159]. Therefore, more empirical evidence is required.

Most resources were designed for adults generally, although a few included programs were designed for specific populations such as farmers, tertiary students, and expectant or new mothers. There were no programs identified in our searches that were designed for hard-to-reach groups such as culturally and linguistically diverse populations or men, and there was only 1 for people from an Aboriginal or Torres Strait Islander cultural background. Web-based interventions provide a useful avenue for administering targeted mental health support to populations who may face barriers to accessing traditional services for cultural reasons. These results highlight the need for more research and development of programs that meet the needs of these at-risk and underserved groups.

\section{Limitations}

The use of a scoring system to evaluate and compare programs was based on a framework for evaluating internet-based interventions [40], as adapted in subsequent research $[15,16]$. This is a useful tool for comparisons across programs and between this research and past studies. However, items may not reflect the overall true value of a program to a particular consumer, with many elements of the program not being included in this scale and not necessarily being quantifiable as a yes or no item. Future research could extend this by examining how consumers and practitioners select an appropriate resource and the factors that they consider to be important in this choice. In addition, although we evaluated the usability of the programs, no completion rates of the included studies were investigated. Future studies should consider examining the completion rate of programs, as program attrition and nonuse attrition are persistent problems with digital interventions [160,161].

At the time of the literature search, there were no national standards for website developers and users to refer to when considering the selection of Australian web-based interventions for mental health. However, on November 30, 2020, the Australian Government released the National Safety and Quality Digital Mental Health Standards for health care providers to refer to when selecting digital media (including web-based programs) for the delivery of high-quality mental health care and suicide prevention (counseling or peer-based support) [162]. The purpose of the National Safety and Quality Digital Mental-Health Standards is to provide consumers and practitioners with information on which to base their selection of trustworthy resources and guide resource developers by providing quality standards. Familiarity and use of the standards, along with the findings from the present research, may help GPs and consumers select the most suitable web-based program for their needs. Although standards are now publicly available, an independent assessment tool to identify where a digital mental health service is meeting the standards or where it can improve is currently being developed and will not be available until late 2021. Similar reviews of this type in the future should include information on whether interventions have met these standards and whether this is a self- or independent assessment.

\section{Comparison With Prior Work}

Overall, of the 52 programs, 9 (17\%) addressed depression, 15 (29\%) addressed anxiety, 13 (25\%) addressed general mental well-being, and 13 (25\%) addressed multiple issues. This is substantially lower than the 32 programs for depression and the 34 programs for anxiety identified by Renton et al [15] and Ashford et al [16], respectively. In part, this is because of the restriction of the current review to programs that were (1) free and (2) accessible to Australians, which excluded several 
currently available international programs such as Beating the Blues or Deprexis, which offer interventions for a fee or are not available to Australians. In addition, the results may highlight the difficulties consumers face in finding suitable programs through search engines [163]. Search engine results were based on the Australian version of Google; however, these results are likely to change over time, as do programs, and the same search conducted at a later date may yield different results. Our search terms were intentionally broad, and we did not require the terms to be used in conjunction with terms describing therapy or self-help. The purpose of broadening the search in this way was to mirror the type of search that a consumer is likely to perform (focused on the issue and not the intervention type).

\section{Conclusions}

A total of 52 web-based programs and web-based programs with downloadable worksheets or resources programs are currently freely available to help Australians with the management of depression, anxiety, suicidal ideation, or general mental well-being. Careful attention is needed by clinicians and consumers to determine whether the interventions they refer to, or access, are evidence-based and considered acceptable by other users, given the varied levels of acceptability and impact. This review complements existing resources by providing website summaries and a clear comparison of website features to inform clinicians and consumers and assist in the selection of the most suitable program for the individual. It also identified important gaps in the availability of free web-based interventions in Australia (eg, for culturally and linguistically diverse populations and based on ACT), which may inform future research and program development initiatives.

\section{Acknowledgments}

The authors would like to acknowledge the program authors who generously gave their time to respond to queries and provided access to their websites. This project was funded by a RACGP Foundation/ANEDGP Innovation Grant 2020 (ANEDGP2020-04).

\section{Authors' Contributions}

This project was overseen by GS and KG. GS and KG devised the concept for the review, with input from CH, NJ, DT, and MS. The grant was applied for and won by GS, CH, KG, NJ, and DT. GS wrote the initial draft. GS, SvdK, and SL conducted the searches. GS, SvdK, SL, and DHB conducted the rapid review. All authors contributed to the final manuscript.

\section{Conflicts of Interest}

The authors KG, DT, and GS are involved in the development and evaluation of ifarmwell (a web-based intervention described in this paper).

\section{Multimedia Appendix 1}

Google search results.

[XLSX File (Microsoft Excel File), 18 KB-Multimedia Appendix 1]

\section{Multimedia Appendix 2}

Program developers and website links.

[DOCX File , 20 KB-Multimedia Appendix 2]

\section{Multimedia Appendix 3}

Program evaluation scores.

[XLSX File (Microsoft Excel File), 16 KB-Multimedia Appendix 3]

\section{Multimedia Appendix 4}

Types of research evaluations of eligible programs and summary of evidence.

[DOCX File, 35 KB-Multimedia Appendix 4]

\section{References}

1. Australia's health 2018. Australian Institute of Health and Welfare. URL: https://www.aihw.gov.au/getmedia/ 7c42913d-295f-4bc9-9c24-4e44eff4a04a/aihw-aus-221.pdf [accessed 2021-12-15]

2. Deaths in Australia. Australian Institute of Health and Welfare. 2021. URL: https://www.aihw.gov.au/reports/phe/229/ deaths/deaths-in-australia/contents/life-expectancy [accessed 2021-12-15]

3. Slade T, Johnston A, Teesson M, Whiteford H, Burgess P, Pirkis J, et al. The Mental Health of Australians 2: Report on the 2007 National Survey of Mental Health and Wellbeing. Canberra: Department of Health and Ageing; 2009. 
4. Luoma JB, Martin CE, Pearson JL. Contact with mental health and primary care providers before suicide: a review of the evidence. Am J Psychiatry 2002 Jun;159(6):909-916 [FREE Full text] [doi: 10.1176/appi.ajp.159.6.909] [Medline: 12042175]

5. Bagayogo IP, Turcios-Wiswe K, Taku K, Peccoralo L, Katz CL. Providing mental health services in the primary care setting: the experiences and perceptions of general practitioners at a New York City clinic. Psychiatr Q 2018 Dec;89(4):897-908. [doi: 10.1007/s11126-018-9587-2] [Medline: 29968148]

6. Wong C, Harrison C, Britt H, Henderson J. Patient use of the internet for health information. Aust Fam Physician 2014 Dec;43(12):875-877. [Medline: 25705739]

7. Household use of information technology. Australian Bureau of Statistics. URL: https://www.abs.gov.au/statistics/industry/ technology-and-innovation/household-use-information-technology/latest-release [accessed 2021-12-15]

8. Barak A, Grohol JM. Current and future trends in internet-supported mental health interventions. J Technol Human Services 2011 Jul;29(3):155-196. [doi: 10.1080/15228835.2011.616939]

9. Hull MJ, Fennell KM, Vallury K, Jones M, Dollman J. A comparison of barriers to mental health support-seeking among farming and non-farming adults in rural South Australia. Aust J Rural Health 2017 Dec;25(6):347-353. [doi: 10.1111/ajr.12352] [Medline: 28618088]

10. McDermott R, Dozois DJ. A randomized controlled trial of internet-delivered CBT and attention bias modification for early intervention of depression. J Experimental Psychopathol 2019 Apr 18;10(2):204380871984250. [doi: $10.1177 / 2043808719842502]$

11. Maher AR, Apaydin EA, Raaen L, Motala A, Baxi S, Hempel S. The use of technology in the clinical care of anxiety: an evidence map. Psychiatr Serv 2021 Feb 01;72(2):195-199. [doi: 10.1176/appi.ps.202000178] [Medline: 33291972]

12. Powell J, Williams V, Atherton H, Bennett K, Yang Y, Davoudianfar M, et al. Effectiveness and cost-effectiveness of a self-guided internet intervention for social anxiety symptoms in a general population sample: randomized controlled trial. J Med Internet Res 2020 Jan 10;22(1):e16804 [FREE Full text] [doi: 10.2196/16804] [Medline: 31821151]

13. Kiropoulos LA, Griffiths KM, Blashki G. Effects of a multilingual information website intervention on the levels of depression literacy and depression-related stigma in Greek-born and Italian-born immigrants living in Australia: a randomized controlled trial. J Med Internet Res 2011 Apr 19;13(2):e34 [FREE Full text] [doi: 10.2196/jmir.1527] [Medline: 21504872]

14. Jorm AF, Korten AE, Jacomb PA, Christensen H, Rodgers B, Pollitt P. "Mental health literacy": a survey of the public's ability to recognise mental disorders and their beliefs about the effectiveness of treatment. Med J Aust 1997 Feb 17;166(4):182-186. [doi: 10.5694/j.1326-5377.1997.tb140071.x] [Medline: 9066546$]$

15. Renton T, Tang H, Ennis N, Cusimano MD, Bhalerao S, Schweizer TA, et al. Web-based intervention programs for depression: a scoping review and evaluation. J Med Internet Res 2014 Sep 23;16(9):e209 [FREE Full text] [doi: 10.2196/jmir.3147] [Medline: 25249003]

16. Ashford MT, Olander EK, Ayers S. Finding web-based anxiety interventions on the world wide web: a scoping review. JMIR Ment Health 2016 Jun 01;3(2):e14 [FREE Full text] [doi: 10.2196/mental.5349] [Medline: 27251763]

17. Griffiths KM, Christensen H, Jorm AF, Evans K, Groves C. Effect of web-based depression literacy and cognitive-behavioural therapy interventions on stigmatising attitudes to depression: randomised controlled trial. Br J Psychiatry 2004 Oct;185:342-349. [doi: 10.1192/bjp.185.4.342] [Medline: 15458995]

18. Kennedy AJ, Brumby SA, Versace VL, Brumby-Rendell T. The ripple effect: a digital intervention to reduce suicide stigma among farming men. BMC Public Health 2020 May 29;20(1):813 [FREE Full text] [doi: 10.1186/s12889-020-08954-5] [Medline: 32471501$]$

19. Kirschner B, Goetzl M, Curtin L. Mental health stigma among college students: test of an interactive online intervention. J Am Coll Health 2020 Oct 13:1-8. [doi: 10.1080/07448481.2020.1826492] [Medline: 33048656]

20. Gunn K, Barrett A, Hughes-Barton D, Turnbull D, Short CE, Brumby S, et al. What farmers want from mental health and wellbeing-focused websites and online interventions. J Rur Stud 2021 Aug;86:298-308. [doi: 10.1016/j.jrurstud.2021.06.016]

21. March S, Spence SH, Donovan CL, Kenardy JA. Large-scale dissemination of internet-based cognitive behavioral therapy for youth anxiety: feasibility and acceptability study. J Med Internet Res 2018 Jul 04;20(7):e234 [FREE Full text] [doi: 10.2196/jmir.9211] [Medline: 29973338]

22. Choi I, Zou J, Titov N, Dear BF, Li S, Johnston L, et al. Culturally attuned internet treatment for depression amongst Chinese Australians: a randomised controlled trial. J Affect Disord 2012 Feb;136(3):459-468. [doi: 10.1016/j.jad.2011.11.003] [Medline: 22177742]

23. Kayrouz R, Dear BF, Karin E, Fogliati VJ, Titov N. A pilot study of a clinician-guided internet-delivered cognitive behavioural therapy for anxiety and depression among Arabs in Australia, presented in both English and Arabic languages. Internet Interv 2016 Sep;5:5-11 [FREE Full text] [doi: 10.1016/j.invent.2016.06.002] [Medline: 30135801]

24. Dear BF, Johnson B, Singh A, Wilkes B, Brkic T, Gupta R, et al. Examining an internet-delivered intervention for anxiety and depression when delivered as a part of routine care for university students: a phase IV trial. J Affect Disord 2019 Sep 01;256:567-577 [FREE Full text] [doi: 10.1016/j.jad.2019.06.044] [Medline: 31280082]

25. Anderson J, O'Moore K, Faraj M, Proudfoot J. Stepped care mental health service in Australian primary care: codesign and feasibility study. Aust. Health Review 2020;44(6):873. [doi: 10.1071/ah19078] 
26. Subotic-Kerry M, King C, O'Moore K, Achilles M, O'Dea B. General practitioners' attitudes toward a web-based mental health service for adolescents: implications for service design and delivery. JMIR Hum Factors 2018 Mar 23;5(1):e12 [FREE Full text] [doi: 10.2196/humanfactors.8913] [Medline: 29572203]

27. Harrer M, Adam SH, Baumeister H, Cuijpers P, Karyotaki E, Auerbach RP, et al. Internet interventions for mental health in university students: a systematic review and meta-analysis. Int J Methods Psychiatr Res 2019 Jun;28(2):e1759 [FREE Full text] [doi: 10.1002/mpr.1759] [Medline: $\underline{30585363}$ ]

28. A guide to digital mental health resources. E-Mental Health in Practice. URL: https://www.emhprac.org.au/resource/ a-guide-to-digital-mental-health-resources/ [accessed 2021-12-15]

29. Köhnen M, Dreier M, Seeralan T, Kriston L, Härter M, Baumeister H, et al. Evidence on technology-based psychological interventions in diagnosed depression: systematic review. JMIR Ment Health 2021 Feb 10;8(2):e21700 [FREE Full text] [doi: 10.2196/21700] [Medline: $\underline{33565981]}$

30. Tokgöz P, Hrynyschyn R, Hafner J, Schönfeld S, Dockweiler C. Digital health interventions in prevention, relapse, and therapy of mild and moderate depression: scoping review. JMIR Ment Health 2021 Apr 16;8(4):e26268 [FREE Full text] [doi: 10.2196/26268] [Medline: 33861201]

31. Rigabert A, Motrico E, Moreno-Peral P, Resurrección DM, Conejo-Cerón S, Cuijpers P, et al. Effectiveness of online psychological and psychoeducational interventions to prevent depression: systematic review and meta-analysis of randomized controlled trials. Clin Psychol Rev 2020 Dec;82:101931. [doi: 10.1016/j.cpr.2020.101931] [Medline: $\underline{33137611]}$

32. Christ C, Schouten MJ, Blankers M, van Schaik DJ, Beekman AT, Wisman MA, et al. Internet and computer-based cognitive behavioral therapy for anxiety and depression in adolescents and young adults: systematic review and meta-analysis. J Med Internet Res 2020 Sep 25;22(9):e17831 [FREE Full text] [doi: 10.2196/17831] [Medline: 32673212]

33. Lehtimaki S, Martic J, Wahl B, Foster KT, Schwalbe N. Evidence on digital mental health interventions for adolescents and young people: systematic overview. JMIR Ment Health 2021 Apr 29;8(4):e25847 [FREE Full text] [doi: 10.2196/25847] [Medline: 33913817$]$

34. Peters MD, Marnie C, Tricco AC, Pollock D, Munn Z, Alexander L, et al. Updated methodological guidance for the conduct of scoping reviews. JBI Evid Synth 2020 Oct;18(10):2119-2126. [doi: 10.11124/JBIES-20-00167] [Medline: 33038124]

35. Tricco AC, Lillie E, Zarin W, O'Brien KK, Colquhoun H, Levac D, et al. PRISMA Extension for Scoping Reviews (PRISMA-ScR): checklist and explanation. Ann Intern Med 2018 Oct 02;169(7):467-473 [FREE Full text] [doi: 10.7326/M18-0850] [Medline: 30178033]

36. Skaczkowski G, van der Kruk S, Gunn K, Howell C, Jensen N, Turnbull D. Scoping review to identify web-based resources for depression, anxiety, suicidal ideation and general wellbeing. Open Science Framework. 2020. URL: https://osf.io/rg9fj [accessed 2022-01-11]

37. Beacon. Beacon 2.0. URL: https://beacon.anu.edu.au/ [accessed 2021-12-15]

38. Head to health. Australian Government Department of Health. URL: https://headtohealth.gov.au/ [accessed 2021-12-15]

39. Resource library. E-Mental Health in Practice. URL: https://www.emhprac.org.au/ [accessed 2021-12-15]

40. Proudfoot J, Klein B, Barak A, Carlbring P, Cuijpers P, Lange A, et al. Establishing guidelines for executing and reporting internet intervention research. Cogn Behav Ther 2011;40(2):82-97. [doi: 10.1080/16506073.2011.573807] [Medline: 25155812]

41. Merlin T, Weston A, Tooher R, Middleton P, Salisbury J, Coleman K. NHMRC levels of evidence and grades for recommendations for developers of guidelines. National Health and Medical Research Council. 2009. URL: https://www. nhmrc.gov.au/sites/default/files/images/NHMRC\%20Levels\%20and\%20Grades\%20(2009).pdf [accessed 2021-12-15]

42. Melvin GA, Gresham D, Beaton S, Coles J, Tonge BJ, Gordon MS, et al. Evaluating the feasibility and effectiveness of an Australian safety planning smartphone application: a pilot study within a tertiary mental health service. Suicide Life Threat Behav 2019 Jun;49(3):846-858 [FREE Full text] [doi: 10.1111/sltb.12490] [Medline: 29999193]

43. Draper M, Rees C, Nathan P. Internet-based self-management of generalised anxiety disorder: a preliminary study. Behav Change 2012 Feb 22;25(4):229-244. [doi: 10.1375/bech.25.4.229]

44. Christensen H, Batterham P, Mackinnon A, Griffiths KM, Kalia Hehir K, Kenardy J, et al. Prevention of generalized anxiety disorder using a web intervention, iChill: randomized controlled trial. J Med Internet Res 2014 Sep 02;16(9):e199 [FREE Full text] [doi: 10.2196/jmir.3507] [Medline: 25270886]

45. Christensen H, Mackinnon AJ, Batterham PJ, O'Dea B, Guastella AJ, Griffiths KM, et al. The effectiveness of an online e-health application compared to attention placebo or Sertraline in the treatment of Generalised Anxiety Disorder. Internet Interventions 2014 Oct;1(4):169-174. [doi: 10.1016/j.invent.2014.08.002]

46. Donker T, Bennett K, Bennett A, Mackinnon A, van Straten A, Cuijpers P, et al. Internet-delivered interpersonal psychotherapy versus internet-delivered cognitive behavioral therapy for adults with depressive symptoms: randomized controlled noninferiority trial. J Med Internet Res 2013 May 13;15(5):e82 [FREE Full text] [doi: 10.2196/jmir.2307] [Medline: 23669884]

47. Crisp D, Griffiths K, Mackinnon A, Bennett K, Christensen H. An online intervention for reducing depressive symptoms: secondary benefits for self-esteem, empowerment and quality of life. Psychiatry Res 2014 Apr 30;216(1):60-66. [doi: 10.1016/j.psychres.2014.01.041] [Medline: 24534125] 
48. Glozier N, Christensen H, Naismith S, Cockayne N, Donkin L, Neal B, et al. Internet-delivered cognitive behavioural therapy for adults with mild to moderate depression and high cardiovascular disease risks: a randomised attention-controlled trial. PLoS One 2013;8(3):e59139. [doi: 10.1371/journal.pone.0059139] [Medline: 23555624]

49. Donkin L, Hickie IB, Christensen H, Naismith SL, Neal B, Cockayne NL, et al. Rethinking the dose-response relationship between usage and outcome in an online intervention for depression: randomized controlled trial. J Med Internet Res 2013 Oct 17;15(10):e231 [FREE Full text] [doi: 10.2196/jmir.2771] [Medline: 24135213]

50. Donker T, Batterham PJ, Warmerdam L, Bennett K, Bennett A, Cuijpers P, et al. Predictors and moderators of response to internet-delivered Interpersonal Psychotherapy and Cognitive Behavior Therapy for depression. J Affect Disord 2013 Oct;151(1):343-351. [doi: 10.1016/j.jad.2013.06.020] [Medline: 23953024]

51. Bowler JO, Mackintosh B, Dunn BD, Mathews A, Dalgleish T, Hoppitt L. A comparison of cognitive bias modification for interpretation and computerized cognitive behavior therapy: effects on anxiety, depression, attentional control, and interpretive bias. J Consult Clin Psychol 2012 Dec;80(6):1021-1033 [FREE Full text] [doi: 10.1037/a0029932] [Medline: 22963595]

52. Farvolden P, Denisoff E, Selby P, Bagby RM, Rudy L. Usage and longitudinal effectiveness of a web-based self-help cognitive behavioral therapy program for panic disorder. J Med Internet Res 2005 Mar 26;7(1):e7 [FREE Full text] [doi: 10.2196/jmir.7.1.e7] [Medline: 15829479]

53. Cunningham JA, Hodgins DC, Toneatto T, Murphy M. A randomized controlled trial of a personalized feedback intervention for problem gamblers. PLoS One 2012;7(2):e31586 [FREE Full text] [doi: 10.1371/journal.pone.0031586] [Medline: 22348112]

54. van Mierlo T, Li X, Hyatt D, Ching AT. Demographic and indication-specific characteristics have limited association with social network engagement: evidence from 24,954 members of four health care support groups. J Med Internet Res 2017 Feb 17;19(2):e40 [FREE Full text] [doi: 10.2196/jmir.6330] [Medline: 28213340]

55. Pittaway S, Cupitt C, Palmer D, Arowobusoye N, Milne R, Holttum S, et al. Comparative, clinical feasibility study of three tools for delivery of cognitive behavioural therapy for mild to moderate depression and anxiety provided on a self-help basis. Ment Health Fam Med 2009 Sep;6(3):145-154 [FREE Full text] [Medline: 22477905]

56. Elison S, Ward J, Williams C, Espie C, Davies G, Dugdale S, et al. Feasibility of a UK community-based, eTherapy mental health service in Greater Manchester: repeated-measures and between-groups study of 'Living Life to the Full Interactive', 'Sleepio' and 'Breaking Free Online' at 'Self Help Services'. BMJ Open 2017 Jul 20;7(7):e016392 [FREE Full text] [doi: 10.1136/bmjopen-2017-016392] [Medline: 28729322]

57. Hadjistavropoulos HD, Pugh NE, Nugent MM, Hesser H, Andersson G, Ivanov M, et al. Therapist-assisted Internet-delivered cognitive behavior therapy for depression and anxiety: translating evidence into clinical practice. J Anxiety Disord 2014 Dec;28(8):884-893 [FREE Full text] [doi: 10.1016/j.janxdis.2014.09.018] [Medline: 25445078]

58. Hadjistavropoulos HD, Pugh NE, Hesser H, Andersson G. Predicting response to therapist-assisted internet-delivered cognitive behavior therapy for depression or anxiety within an open dissemination trial. Behav Ther 2016 Mar;47(2):155-165. [doi: 10.1016/j.beth.2015.10.006] [Medline: 26956649]

59. Al-Asadi AM, Klein B, Meyer D. Posttreatment attrition and its predictors, attrition bias, and treatment efficacy of the anxiety online programs. J Med Internet Res 2014 Oct 14;16(10):e232 [FREE Full text] [doi: 10.2196/jmir.3513] [Medline: 25316533]

60. Klein B, Meyer D, Austin DW, Kyrios M. Anxiety online: a virtual clinic: preliminary outcomes following completion of five fully automated treatment programs for anxiety disorders and symptoms. J Med Internet Res 2011 Nov 04;13(4):e89 [FREE Full text] [doi: 10.2196/jmir.1918] [Medline: 22057287]

61. Titov N, Schofield C, Staples L, Dear BF, Nielssen O. A comparison of Indigenous and non-Indigenous users of MindSpot: an Australian digital mental health service. Australas Psychiatry 2019 Aug;27(4):352-357. [doi: 10.1177/1039856218789784] [Medline: $\underline{30058351]}$

62. Titov N, Dear BF, Johnston L, McEvoy PM, Wootton B, Terides MD, et al. Improving adherence and clinical outcomes in self-guided internet treatment for anxiety and depression: a 12-month follow-up of a randomised controlled trial. PLoS One 2014;9(2):e89591 [FREE Full text] [doi: 10.1371/journal.pone.0089591] [Medline: 24586897]

63. Dear BF, Fogliati VJ, Fogliati R, Johnson B, Boyle O, Karin E, et al. Treating anxiety and depression in young adults: a randomised controlled trial comparing clinician-guided versus self-guided Internet-delivered cognitive behavioural therapy. Aust N Z J Psychiatry 2018 Jul;52(7):668-679. [doi: 10.1177/0004867417738055] [Medline: 29064283]

64. Lee Y, Gao L, Dear BF, Titov N, Mihalopoulos C. The cost-effectiveness of the online MindSpot clinic for the treatment of depression and anxiety in Australia. J Ment Health Policy Econ 2017 Dec 01;20(4):155-166. [Medline: 29300702]

65. Kirkpatrick T, Manoukian L, Dear B, Johnston L, Titov N. A feasibility open trial of internet-delivered cognitive-behavioural therapy (iCBT) among consumers of a non-governmental mental health organisation with anxiety. PeerJ 2013;1:e210 [FREE Full text] [doi: 10.7717/peerj.210] [Medline: 24349897]

66. Fogliati VJ, Dear BF, Staples LG, Terides MD, Sheehan J, Johnston L, et al. Disorder-specific versus transdiagnostic and clinician-guided versus self-guided internet-delivered treatment for panic disorder and comorbid disorders: a randomized controlled trial. J Anxiety Disord 2016 Apr;39:88-102 [FREE Full text] [doi: 10.1016/j.janxdis.2016.03.005] [Medline: $\underline{27003376]}$ 
67. Titov N, Dear BF, Staples LG, Bennett-Levy J, Klein B, Rapee RM, et al. MindSpot clinic: an accessible, efficient, and effective online treatment service for anxiety and depression. Psychiatr Serv 2015 Oct;66(10):1043-1050. [doi: 10.1176/appi.ps.201400477] [Medline: 26130001]

68. Titov N, Dear BF, Johnston L, Lorian C, Zou J, Wootton B, et al. Improving adherence and clinical outcomes in self-guided internet treatment for anxiety and depression: randomised controlled trial. PLoS One 2013;8(7):e62873 [FREE Full text] [doi: 10.1371/journal.pone.0062873] [Medline: 23843932]

69. Dear BF, Staples LG, Terides MD, Fogliati VJ, Sheehan J, Johnston L, et al. Transdiagnostic versus disorder-specific and clinician-guided versus self-guided internet-delivered treatment for Social Anxiety Disorder and comorbid disorders: a randomized controlled trial. J Anxiety Disord 2016 Aug;42:30-44 [FREE Full text] [doi: 10.1016/j.janxdis.2016.05.004] [Medline: 27261562]

70. Dear BF, Staples LG, Terides MD, Karin E, Zou J, Johnston L, et al. Transdiagnostic versus disorder-specific and clinician-guided versus self-guided internet-delivered treatment for generalized anxiety disorder and comorbid disorders: a randomized controlled trial. J Anxiety Disord 2015 Dec;36:63-77 [FREE Full text] [doi: 10.1016/j.janxdis.2015.09.003] [Medline: 26460536$]$

71. Titov N, Andrews G, Johnston L, Robinson E, Spence J. Transdiagnostic internet treatment for anxiety disorders: a randomized controlled trial. Behav Res Ther 2010 Sep;48(9):890-899. [doi: 10.1016/j.brat.2010.05.014] [Medline: 20561606]

72. Titov N, Dear BF, Schwencke G, Andrews G, Johnston L, Craske MG, et al. Transdiagnostic internet treatment for anxiety and depression: a randomised controlled trial. Behav Res Ther 2011 Aug;49(8):441-452. [doi: 10.1016/j.brat.2011.03.007] [Medline: 21679925]

73. Johnston L, Titov N, Andrews G, Dear BF, Spence J. Comorbidity and internet-delivered transdiagnostic cognitive behavioural therapy for anxiety disorders. Cogn Behav Ther 2013;42(3):180-192. [doi: 10.1080/16506073.2012.753108] [Medline: 23458319]

74. Johnston L, Titov N, Andrews G, Spence J, Dear BF. A RCT of a transdiagnostic internet-delivered treatment for three anxiety disorders: examination of support roles and disorder-specific outcomes. PLoS One 2011;6(11):e28079 [FREE Full text] [doi: 10.1371/journal.pone.0028079] [Medline: 22132216]

75. Dear BF, Zou JB, Ali S, Lorian CN, Johnston L, Sheehan J, et al. Clinical and cost-effectiveness of therapist-guided internet-delivered cognitive behavior therapy for older adults with symptoms of anxiety: a randomized controlled trial. Behav Ther 2015 Mar;46(2):206-217. [doi: 10.1016/j.beth.2014.09.007] [Medline: 25645169]

76. Titov N, Dear BF, Ali S, Zou JB, Lorian CN, Johnston L, et al. Clinical and cost-effectiveness of therapist-guided internet-delivered cognitive behavior therapy for older adults with symptoms of depression: a randomized controlled trial. Behav Ther 2015 Mar;46(2):193-205. [doi: 10.1016/j.beth.2014.09.008] [Medline: 25645168]

77. Zou JB, Dear BF, Titov N, Lorian CN, Johnston L, Spence J, et al. Brief internet-delivered cognitive behavioral therapy for anxiety in older adults: a feasibility trial. J Anxiety Disord 2012 Aug;26(6):650-655. [doi: 10.1016/j.janxdis.2012.04.002] [Medline: 22659078]

78. Staples LG, Fogliati VJ, Dear BF, Nielssen O, Titov N. Internet-delivered treatment for older adults with anxiety and depression: implementation of the Wellbeing Plus Course in routine clinical care and comparison with research trial outcomes. BJPsych Open 2016 Sep;2(5):307-313 [FREE Full text] [doi: 10.1192/bjpo.bp.116.003400] [Medline: 27703794]

79. Titov N, Fogliati VJ, Staples LG, Gandy M, Johnston L, Wootton B, et al. Treating anxiety and depression in older adults: randomised controlled trial comparing guided v. self-guided internet-delivered cognitive-behavioural therapy. BJPsych Open 2016 Feb 1;2(1):50-58 [FREE Full text] [doi: 10.1192/bjpo.bp.115.002139] [Medline: 27703754]

80. Read J, Sharpe L, Burton AL, Arean PA, Raue PJ, McDonald S, et al. A randomized controlled trial of internet-delivered cognitive behaviour therapy to prevent the development of depressive disorders in older adults with multimorbidity. J Affect Disord 2020 Mar 01;264:464-473. [doi: 10.1016/j.jad.2019.11.077] [Medline: 31767215]

81. Phillips R, Schneider J, Molosankwe I, Leese M, Foroushani PS, Grime P, et al. Randomized controlled trial of computerized cognitive behavioural therapy for depressive symptoms: effectiveness and costs of a workplace intervention. Psychol Med 2014 Mar;44(4):741-752 [FREE Full text] [doi: 10.1017/S0033291713001323] [Medline: 23795621]

82. Christensen H, Griffiths KM, Korten AE, Brittliffe K, Groves C. A comparison of changes in anxiety and depression symptoms of spontaneous users and trial participants of a cognitive behavior therapy website. J Med Internet Res 2004 Dec 22;6(4):e46 [FREE Full text] [doi: 10.2196/jmir.6.4.e46] [Medline: 15631970]

83. Mackinnon A, Griffiths KM, Christensen H. Comparative randomised trial of online cognitive-behavioural therapy and an information website for depression: 12-month outcomes. Br J Psychiatry 2008 Feb;192(2):130-134. [doi:

10.1192/bjp.bp.106.032078] [Medline: 18245031]

84. Christensen H, Griffiths KM, Korten A. Web-based cognitive behavior therapy: analysis of site usage and changes in depression and anxiety scores. J Med Internet Res 2002;4(1):e3 [FREE Full text] [doi: 10.2196/jmir.4.1.e3] [Medline: 11956035]

85. Calear AL, Christensen H, Mackinnon A, Griffiths KM, O'Kearney R. The YouthMood Project: a cluster randomized controlled trial of an online cognitive behavioral program with adolescents. J Consult Clin Psychol 2009 Dec;77(6):1021-1032. [doi: 10.1037/a0017391] [Medline: 19968379] 
86. Christensen H, Griffiths KM, Mackinnon AJ, Brittliffe K. Online randomized controlled trial of brief and full cognitive behaviour therapy for depression. Psychol Med 2006 Dec;36(12):1737-1746. [doi: 10.1017/S0033291706008695] [Medline: $\underline{16938144]}$

87. Ellis L, Campbell A, Sethi S, O'Dea B. Comparative randomized trial of an online Cognitive-Behavioral Therapy program and an online support group for depression and anxiety. J Cyber Ther Rehab 2011;4(4):461-467 [FREE Full text]

88. Farrer L, Christensen H, Griffiths KM, Mackinnon A. Internet-based CBT for depression with and without telephone tracking in a national helpline: randomised controlled trial. PLoS One 2011;6(11):e28099 [FREE Full text] [doi: 10.1371/journal.pone.0028099] [Medline: 22140514]

89. Hickie IB, Davenport TA, Luscombe GM, Moore M, Griffiths KM, Christensen H. Practitioner-supported delivery of internet-based cognitive behaviour therapy: evaluation of the feasibility of conducting a cluster randomised trial. Med $\mathbf{J}$ Aust 2010 Jun 07;192(S11):S31-S35. [doi: 10.5694/j.1326-5377.2010.tb03690.x] [Medline: 20528705]

90. Sethi S, Campbell AJ, Ellis LA. The use of computerized self-help packages to treat adolescent depression and anxiety. J Technol Human Serv 2010 Aug 31;28(3):144-160. [doi: 10.1080/15228835.2010.508317]

91. Lintvedt OK, Griffiths KM, Sørensen K, Østvik AR, Wang CE, Eisemann M, et al. Evaluating the effectiveness and efficacy of unguided internet-based self-help intervention for the prevention of depression: a randomized controlled trial. Clin Psychol Psychother 2013;20(1):10-27. [doi: 10.1002/cpp.770] [Medline: 21887811]

92. Calear AL, Christensen H, Mackinnon A, Griffiths KM. Adherence to the MoodGYM program: outcomes and predictors for an adolescent school-based population. J Affect Disord 2013 May;147(1-3):338-344. [doi: 10.1016/j.jad.2012.11.036] [Medline: 23245469]

93. Gilbody S, Littlewood E, Hewitt C, Brierley G, Tharmanathan P, Araya R, REEACT Team. Computerised cognitive behaviour therapy (cCBT) as treatment for depression in primary care (REEACT trial): large scale pragmatic randomised controlled trial. BMJ 2015 Nov 11;351:h5627 [FREE Full text] [doi: 10.1136/bmj.h5627] [Medline: 26559241]

94. Høifødt RS, Lillevoll KR, Griffiths KM, Wilsgaard T, Eisemann M, Waterloo K, et al. The clinical effectiveness of web-based cognitive behavioral therapy with face-to-face therapist support for depressed primary care patients: randomized controlled trial. J Med Internet Res 2013 Aug 05;15(8):e153 [FREE Full text] [doi: 10.2196/jmir.2714] [Medline: 23916965]

95. Powell J, Hamborg T, Stallard N, Burls A, McSorley J, Bennett K, et al. Effectiveness of a web-based cognitive-behavioral tool to improve mental well-being in the general population: randomized controlled trial. J Med Internet Res 2012 Dec 31;15(1):e2 [FREE Full text] [doi: 10.2196/jmir.2240] [Medline: 23302475]

96. Sethi S. Treating youth depression and anxiety: a randomised controlled trial examining the efficacy of computerised versus face - to - face cognitive behaviour therapy. Aust Psychol 2020 Nov 12;48(4):249-257. [doi: 10.1111/ap.12006]

97. Twomey C, O'Reilly G, Byrne M, Bury M, White A, Kissane S, et al. A randomized controlled trial of the computerized CBT programme, MoodGYM, for public mental health service users waiting for interventions. Br J Clin Psychol 2014 Nov;53(4):433-450. [doi: 10.1111/bjc.12055] [Medline: 24831119]

98. Howell AN, Rheingold AA, Uhde TW, Guille C. Web-based CBT for the prevention of anxiety symptoms among medical and health science graduate students. Cogn Behav Ther 2019 Sep;48(5):385-405 [FREE Full text] [doi: 10.1080/16506073.2018.1533575] [Medline: 30526364]

99. Löbner M, Pabst A, Stein J, Dorow M, Matschinger H, Luppa M, et al. Computerized cognitive behavior therapy for patients with mild to moderately severe depression in primary care: a pragmatic cluster randomized controlled trial (@ktiv). J Affect Disord 2018 Oct 01;238:317-326. [doi: 10.1016/j.jad.2018.06.008] [Medline: 29902736]

100. O'Kearney R, Gibson M, Christensen H, Griffiths KM. Effects of a cognitive-behavioural internet program on depression, vulnerability to depression and stigma in adolescent males: a school-based controlled trial. Cogn Behav Ther 2006;35(1):43-54. [doi: 10.1080/16506070500303456] [Medline: 16500776]

101. O'Kearney R, Kang K, Christensen H, Griffiths K. A controlled trial of a school-based internet program for reducing depressive symptoms in adolescent girls. Depress Anxiety 2009;26(1):65-72. [doi: 10.1002/da.20507] [Medline: 18828141]

102. Christensen H, Griffiths K, Groves C, Korten A. Free range users and one hit wonders: community users of an internet-based cognitive behaviour therapy program. Aust N Z J Psychiatry 2006 Jan;40(1):59-62. [doi: 10.1080/j.1440-1614.2006.01743.x] [Medline: 16403040]

103. Danaher BG, Milgrom J, Seeley JR, Stuart S, Schembri C, Tyler MS, et al. Web-based intervention for postpartum depression: formative research and design of the MomMoodBooster program. JMIR Res Protoc 2012 Nov 22;1(2):e18 [FREE Full text] [doi: 10.2196/resprot.2329] [Medline: 23612274]

104. Danaher BG, Milgrom J, Seeley JR, Stuart S, Schembri C, Tyler MS, et al. MomMoodBooster web-based intervention for postpartum depression: feasibility trial results. J Med Internet Res 2013 Nov 04;15(11):e242 [FREE Full text] [doi: 10.2196/jmir.2876] [Medline: 24191345]

105. Milgrom J, Danaher BG, Gemmill AW, Holt C, Holt CJ, Seeley JR, et al. Internet cognitive behavioral therapy for women with postnatal depression: a randomized controlled trial of MumMoodBooster. J Med Internet Res 2016 Mar 07;18(3):e54 [FREE Full text] [doi: 10.2196/jmir.4993] [Medline: 26952645]

106. Clarke J, Proudfoot J, Ma H. Mobile phone and web-based cognitive behavior therapy for depressive symptoms and mental health comorbidities in people living with diabetes: results of a feasibility study. JMIR Ment Health 2016 May 31;3(2):e23 [FREE Full text] [doi: 10.2196/mental.5131] [Medline: 27245948] 
107. Harrison V, Proudfoot J, Wee PP, Parker G, Pavlovic DH, Manicavasagar V. Mobile mental health: review of the emerging field and proof of concept study. J Ment Health 2011 Dec;20(6):509-524. [doi: 10.3109/09638237.2011.608746] [Medline: 21988230]

108. Proudfoot J, Clarke J, Birch M, Whitton AE, Parker G, Manicavasagar V, et al. Impact of a mobile phone and web program on symptom and functional outcomes for people with mild-to-moderate depression, anxiety and stress: a randomised controlled trial. BMC Psychiatry 2013 Nov 18;13:312 [FREE Full text] [doi: 10.1186/1471-244X-13-312] [Medline: 24237617]

109. Clarke J, Proudfoot J, Birch M, Whitton AE, Parker G, Manicavasagar V, et al. Effects of mental health self-efficacy on outcomes of a mobile phone and web intervention for mild-to-moderate depression, anxiety and stress: secondary analysis of a randomised controlled trial. BMC Psychiatry 2014 Sep 26;14:272 [FREE Full text] [doi: 10.1186/s12888-014-0272-1] [Medline: 25252853]

110. Baldwin PA, Sanatkar S, Clarke J, Fletcher S, Gunn J, Wilhelm K, et al. A web-based mental health intervention to improve social and occupational functioning in adults with type 2 diabetes (the springboard trial): 12-month outcomes of a randomized controlled trial. J Med Internet Res 2020 Dec 01;22(12):e16729 [FREE Full text] [doi: 10.2196/16729] [Medline: 33258790]

111. Newby JM, Mewton L, Andrews G. Transdiagnostic versus disorder-specific internet-delivered cognitive behaviour therapy for anxiety and depression in primary care. J Anxiety Disord 2017 Mar;46:25-34. [doi: 10.1016/j.janxdis.2016.06.002] [Medline: 27396841]

112. Sunderland M, Wong N, Hilvert-Bruce Z, Andrews G. Investigating trajectories of change in psychological distress amongst patients with depression and generalised anxiety disorder treated with internet cognitive behavioural therapy. Behav Res Ther 2012 Jun;50(6):374-380. [doi: 10.1016/j.brat.2012.03.005] [Medline: 22498311]

113. Perini S, Titov N, Andrews G. The Climate Sadness program: an open trial of internet-based treatment for depression. E J Applied Psychol 2008 Dec 23;4(2):18-24. [doi: 10.7790/ejap.v4i2.135]

114. Williams AD, Andrews G. The effectiveness of internet cognitive behavioural therapy (iCBT) for depression in primary care: a quality assurance study. PLoS One 2013;8(2):e57447 [FREE Full text] [doi: 10.1371/journal.pone.0057447] [Medline: 23451231]

115. Watts S, Newby JM, Mewton L, Andrews G. A clinical audit of changes in suicide ideas with internet treatment for depression. BMJ Open 2012;2(5):e001558 [FREE Full text] [doi: 10.1136/bmjopen-2012-001558] [Medline: 22983787]

116. Mewton L, Andrews G. Cognitive behaviour therapy via the internet for depression: a useful strategy to reduce suicidal ideation. J Affect Disord 2015 Jan 01;170:78-84. [doi: 10.1016/j.jad.2014.08.038] [Medline: 25233243]

117. Hobbs MJ, Joubert AE, Mahoney AE, Andrews G. Treating late-life depression: comparing the effects of internet-delivered cognitive behavior therapy across the adult lifespan. J Affect Disord 2018 Jan 15;226:58-65. [doi: 10.1016/j.jad.2017.09.026] [Medline: 28963865]

118. Perini S, Titov N, Andrews G. Clinician-assisted internet-based treatment is effective for depression: randomized controlled trial. Aust N Z J Psychiatry 2009 Jun;43(6):571-578. [doi: 10.1080/00048670902873722] [Medline: 19440890]

119. Titov N, Andrews G, Davies M, McIntyre K, Robinson E, Solley K. Internet treatment for depression: a randomized controlled trial comparing clinician vs. technician assistance. PLoS One 2010 Jun 08;5(6):e10939 [FREE Full text] [doi: 10.1371/journal.pone.0010939] [Medline: 20544030]

120. Rosso IM, Killgore WD, Olson EA, Webb CA, Fukunaga R, Auerbach RP, et al. Internet-based cognitive behavior therapy for major depressive disorder: a randomized controlled trial. Depress Anxiety 2017 Mar;34(3):236-245 [FREE Full text] [doi: 10.1002/da.22590] [Medline: 28009467]

121. Smith J, Newby JM, Burston N, Murphy MJ, Michael S, Mackenzie A, et al. Help from home for depression: a randomised controlled trial comparing internet-delivered cognitive behaviour therapy with bibliotherapy for depression. Internet Interv 2017 Sep;9:25-37 [FREE Full text] [doi: 10.1016/j.invent.2017.05.001] [Medline: 30135834]

122. Watts S, Mackenzie A, Thomas C, Griskaitis A, Mewton L, Williams A, et al. CBT for depression: a pilot RCT comparing mobile phone vs. computer. BMC Psychiatry 2013 Feb 07;13:49 [FREE Full text] [doi: 10.1186/1471-244X-13-49] [Medline: 23391304]

123. O'moore KA, Newby JM, Andrews G, Hunter DJ, Bennell K, Smith J, et al. Internet cognitive-behavioral therapy for depression in older adults with knee osteoarthritis: a randomized controlled trial. Arthritis Care Res (Hoboken) 2018 Jan;70(1):61-70. [doi: 10.1002/acr.23257] [Medline: 28426917]

124. Fletcher S, Chondros P, Densley K, Murray E, Dowrick C, Coe A, et al. Matching depression management to severity prognosis in primary care: results of the Target-D randomised controlled trial. Br J Gen Pract 2021;71(703):85-94 [FREE Full text] [doi: 10.3399/BJGP.2020.0783] [Medline: 33431380]

125. Hilvert-Bruce Z, Rossouw PJ, Wong N, Sunderland M, Andrews G. Adherence as a determinant of effectiveness of internet cognitive behavioural therapy for anxiety and depressive disorders. Behav Res Ther 2012 Aug;50(7-8):463-468. [doi: 10.1016/j.brat.2012.04.001] [Medline: 22659155]

126. Newby JM, Mahoney AE, Mason EC, Smith J, Uppal S, Andrews G. Pilot trial of a therapist-supported internet-delivered cognitive behavioural therapy program for health anxiety. Internet Interv 2016 Nov;6:71-79 [FREE Full text] [doi: 10.1016/j.invent.2016.09.007] [Medline: $\underline{30135816]}$ 
127. Newby JM, Haskelberg H, Hobbs MJ, Mahoney AE, Mason E, Andrews G. The effectiveness of internet-delivered cognitive behavioural therapy for health anxiety in routine care. J Affect Disord 2020 Mar 01;264:535-542. [doi: 10.1016/j.jad.2019.11.087] [Medline: $\underline{\text { 31780130] }}$

128. Newby JM, Smith J, Uppal S, Mason E, Mahoney AE, Andrews G. Internet-based cognitive behavioral therapy versus psychoeducation control for illness anxiety disorder and somatic symptom disorder: a randomized controlled trial. J Consult Clin Psychol 2018 Jan;86(1):89-98. [doi: 10.1037/ccp0000248] [Medline: 29172593]

129. Kladnitski N, Smith J, Allen A, Andrews G, Newby JM. Online mindfulness-enhanced cognitive behavioural therapy for anxiety and depression: outcomes of a pilot trial. Internet Interv 2018 Sep;13:41-50 [FREE Full text] [doi: 10.1016/j.invent.2018.06.003] [Medline: 30206518]

130. Kladnitski N, Smith J, Uppal S, James MA, Allen AR, Andrews G, et al. Transdiagnostic internet-delivered CBT and mindfulness-based treatment for depression and anxiety: a randomised controlled trial. Internet Interv 2020 Apr;20:100310 [FREE Full text] [doi: 10.1016/j.invent.2020.100310] [Medline: 32140427]

131. Newby JM, Mewton L, Williams AD, Andrews G. Effectiveness of transdiagnostic internet cognitive behavioural treatment for mixed anxiety and depression in primary care. J Affect Disord 2014 Aug;165:45-52. [doi: 10.1016/j.jad.2014.04.037] [Medline: 24882176]

132. Morgan C, Mason E, Newby JM, Mahoney AE, Hobbs MJ, McAloon J, et al. The effectiveness of unguided internet cognitive behavioural therapy for mixed anxiety and depression. Internet Interv 2017 Dec;10:47-53 [FREE Full text] [doi: 10.1016/j.invent.2017.10.003] [Medline: 30135752]

133. Mahoney AE, Newby JM, Hobbs MJ, Williams AD, Andrews G. Reducing behavioral avoidance with internet-delivered cognitive behavior therapy for generalized anxiety disorder. Internet Interv 2019 Mar;15:105-109 [FREE Full text] [doi: 10.1016/j.invent.2017.11.004] [Medline: $\underline{30792961]}$

134. Newby JM, Mackenzie A, Williams AD, McIntyre K, Watts S, Wong N, et al. Internet cognitive behavioural therapy for mixed anxiety and depression: a randomized controlled trial and evidence of effectiveness in primary care. Psychol Med 2013 Dec;43(12):2635-2648. [doi: 10.1017/S0033291713000111] [Medline: 23419552]

135. Loughnan SA, Sie A, Hobbs MJ, Joubert AE, Smith J, Haskelberg H, et al. A randomized controlled trial of 'MUMentum Pregnancy': internet-delivered cognitive behavioral therapy program for antenatal anxiety and depression. J Affect Disord 2019 Jan 15;243:381-390. [doi: 10.1016/j.jad.2018.09.057] [Medline: 30266030]

136. Loughnan SA, Butler C, Sie AA, Grierson AB, Chen AZ, Hobbs MJ, et al. A randomised controlled trial of 'MUMentum postnatal': internet-delivered cognitive behavioural therapy for anxiety and depression in postpartum women. Behav Res Ther 2019 May;116:94-103. [doi: 10.1016/j.brat.2019.03.001] [Medline: 30877878]

137. Wims E, Titov N, Andrews G. The Climate Panic program: an open trial of internet-based treatment for panic disorder. E J Applied Psychol 2008 Dec 23;4(2). [doi: 10.7790/ejap.v4i2.134]

138. Bell CJ, Colhoun HC, Carter FA, Frampton CM. Effectiveness of computerised cognitive behaviour therapy for anxiety disorders in secondary care. Aust N Z J Psychiatry 2012 Jul;46(7):630-640. [doi: 10.1177/0004867412437345] [Medline: 22327097]

139. Wims E, Titov N, Andrews G, Choi I. Clinician-assisted Internet-based treatment is effective for panic: a randomized controlled trial. Aust N Z J Psychiatry 2010 Jul;44(7):599-607. [doi: 10.3109/00048671003614171] [Medline: 20560847]

140. Allen AR, Newby JM, Mackenzie A, Smith J, Boulton M, Loughnan SA, et al. Internet cognitive-behavioural treatment for panic disorder: randomised controlled trial and evidence of effectiveness in primary care. BJPsych Open 2016 Mar;2(2):154-162 [FREE Full text] [doi: 10.1192/bjpo.bp.115.001826] [Medline: 27703768]

141. Aydos L, Titov N, Andrews G. Shyness 5: the clinical effectiveness of Internet-based clinician-assisted treatment of social phobia. Australas Psychiatry 2009 Dec;17(6):488-492. [doi: 10.1080/10398560903284943] [Medline: 20001373]

142. Titov N, Andrews G, Schwencke G, Drobny J, Einstein D. Shyness 1: distance treatment of social phobia over the internet. Aust N Z J Psychiatry 2008 Jul;42(7):585-594. [doi: 10.1080/00048670802119762] [Medline: 18612862]

143. Titov N, Andrews G, Schwencke G. Shyness 2: treating social phobia online: replication and extension. Aust N Z J Psychiatry 2008 Jul;42(7):595-605. [doi: 10.1080/00048670802119820] [Medline: 18612863]

144. Titov N, Andrews G, Choi I, Schwencke G, Mahoney A. Shyness 3: randomized controlled trial of guided versus unguided internet-based CBT for social phobia. Aust N Z J Psychiatry 2008 Dec;42(12):1030-1040. [doi: 10.1080/00048670802512107] [Medline: 19016091]

145. Andrews G, Davies M, Titov N. Effectiveness randomized controlled trial of face to face versus internet cognitive behaviour therapy for social phobia. Aust N Z J Psychiatry 2011 Apr;45(4):337-340. [doi: 10.3109/00048674.2010.538840] [Medline: 21323490]

146. Titov N, Gibson M, Andrews G, McEvoy P. Internet treatment for social phobia reduces comorbidity. Aust N Z J Psychiatry 2009 Aug;43(8):754-759. [doi: 10.1080/00048670903001992] [Medline: 19629797]

147. Mewton L, Wong N, Andrews G. The effectiveness of internet cognitive behavioural therapy for generalized anxiety disorder in clinical practice. Depress Anxiety 2012 Oct;29(10):843-849. [doi: 10.1002/da.21995] [Medline: 22949296]

148. Hobbs MJ, Mahoney AE, Andrews G. Integrating iCBT for generalized anxiety disorder into routine clinical care: treatment effects across the adult lifespan. J Anxiety Disord 2017 Oct;51:47-54. [doi: 10.1016/j.janxdis.2017.09.003] [Medline: $\underline{28926805]}$ 
149. Robinson E, Titov N, Andrews G, McIntyre K, Schwencke G, Solley K. Internet treatment for generalized anxiety disorder: a randomized controlled trial comparing clinician vs. technician assistance. PLoS One 2010 Jun 03;5(6):e10942 [FREE Full text] [doi: 10.1371/journal.pone.0010942] [Medline: 20532167]

150. Titov N, Andrews G, Robinson E, Schwencke G, Johnston L, Solley K, et al. Clinician-assisted internet-based treatment is effective for generalized anxiety disorder: randomized controlled trial. Aust N Z J Psychiatry 2009 Jan 01;43(10):905-912. [doi: 10.1080/00048670903179269]

151. Lorian CN, Titov N, Grisham JR. Changes in risk-taking over the course of an internet-delivered cognitive behavioral therapy treatment for generalized anxiety disorder. J Anxiety Disord 2012 Jan;26(1):140-149. [doi: 10.1016/j.janxdis.2011.10.003] [Medline: 22079215]

152. Hayes SC, Pistorello J, Levin ME. Acceptance and commitment therapy as a unified model of behavior change. Counsel Psychol 2012 Sep 26;40(7):976-1002. [doi: 10.1177/0011000012460836]

153. Thompson EM, Destree L, Albertella L, Fontenelle LF. Internet-based acceptance and commitment therapy: a transdiagnostic systematic review and meta-analysis for mental health outcomes. Behav Ther 2021 Mar;52(2):492-507. [doi: 10.1016/j.beth.2020.07.002] [Medline: 33622516]

154. Wells A, Carter K. Generalized anxiety disorder. In: Carr A, McNulty M, editors. The Handbook of Adult Clinical Psychology. Abingdon: Routledge; 2016:447-481.

155. Gross J, Thompson R. Emotion regulation: conceptual foundations. In: Gross JJ, editor. Handbook of Emotion Regulation. New York: The Guilford Press; 2007:3-24.

156. Peprah K, Argáez C. Dialectical Behavioral Therapy for Adults with Mental Illness: A Review of Clinical Effectiveness and Guidelines. Ottawa (ON): Canadian Agency for Drugs and Technologies in Health; 2017.

157. Danaher BG, McKay HG, Seeley JR. The information architecture of behavior change websites. J Med Internet Res 2005 May 18;7(2):e12 [FREE Full text] [doi: 10.2196/jmir.7.2.e12] [Medline: 15914459]

158. Pugatch J, Grenen E, Surla S, Schwarz M, Cole-Lewis H. Information architecture of web-based interventions to improve health outcomes: systematic review. J Med Internet Res 2018 Mar 21;20(3):e97 [FREE Full text] [doi: 10.2196/jmir.7867] [Medline: 29563076]

159. Norman P, Webb TL, Millings A, Pechey L. Does the structure (tunneled vs. free-roam) and content (if-then plans vs. choosing strategies) of a brief online alcohol intervention effect engagement and effectiveness? A randomized controlled trial. Transl Behav Med 2019 Nov 25;9(6):1122-1130. [doi: 10.1093/tbm/ibz110] [Medline: 31287897]

160. Mohr DC, Burns MN, Schueller SM, Clarke G, Klinkman M. Behavioral intervention technologies: evidence review and recommendations for future research in mental health. Gen Hosp Psychiatry 2013;35(4):332-338 [FREE Full text] [doi: 10.1016/j.genhosppsych.2013.03.008] [Medline: 23664503]

161. Mohr DC, Cuijpers P, Lehman K. Supportive accountability: a model for providing human support to enhance adherence to eHealth interventions. J Med Internet Res 2011 Mar 10;13(1):e30 [FREE Full text] [doi: 10.2196/jmir.1602] [Medline: 21393123]

162. New standards open the door to safe and effective digital mental health care. Australian Commission on Safety and Quality in Healthcare. 2020. URL: https://www.safetyandquality.gov.au/publications-and-resources/media-releases/ new-standards-open-door-safe-and-effective-digital-mental-health-care [accessed 2021-12-15]

163. Christensen H, Griffiths K. The internet and mental health literacy. Aust N Z J Psychiatry 2000 Dec;34(6):975-979. [doi: 10.1080/000486700272] [Medline: 11127628]

\section{Abbreviations}

ACT: acceptance and commitment therapy

CBT: cognitive behavioral therapy

CCI: Centre for Clinical Interventions

DBT: dialectical behavior therapy

eMHPrac: e-Mental Health in Practice

GP: general practitioner

LGBQ: lesbian, gay, bisexual, and queer

PRISMA-ScR: Preferred Reporting Items for Systematic Reviews and Meta-Analyses extension for Scoping Reviews

RCT: randomized controlled trial 
Edited by J Torous; submitted 07.06.21; peer-reviewed by H Haskelberg, $R$ Kessler; comments to author 17.07.21; revised version received 23.07.21; accepted 12.08.21; published 08.02.22

Please cite as:

Skaczkowski G, van der Kruk S, Loxton S, Hughes-Barton D, Howell C, Turnbull D, Jensen N, Smout M, Gunn K

Web-Based Interventions to Help Australian Adults Address Depression, Anxiety, Suicidal Ideation, and General Mental Well-being:

Scoping Review

JMIR Ment Health 2022;9(2):e31018

URL: https://mental.jmir.org/2022/2/e31018

doi: $10.2196 / 31018$

PMID:

(C) Gemma Skaczkowski, Shannen van der Kruk, Sophie Loxton, Donna Hughes-Barton, Cate Howell, Deborah Turnbull, Neil Jensen, Matthew Smout, Kate Gunn. Originally published in JMIR Mental Health (https://mental.jmir.org), 08.02.2022. This is an open-access article distributed under the terms of the Creative Commons Attribution License (https://creativecommons.org/licenses/by/4.0/), which permits unrestricted use, distribution, and reproduction in any medium, provided the original work, first published in JMIR Mental Health, is properly cited. The complete bibliographic information, a link to the original publication on https://mental.jmir.org/, as well as this copyright and license information must be included. 